\title{
Differential yet integral contributions of Nrf1 and Nrf2 in the human antioxidant cytoprotective response against tert-butylhydroquinone as a pro-oxidative stressor \\ Reziyamu Wufur, Zhuo Fan, Keli Liu, and Yiguo Zhang*
}

The Laboratory of Cell Biochemistry and Topogenetic Regulation, College of Bioengineering, Chongqing University, No. 174 Shazheng Street, Shapingba District, Chongqing 400044, China.

*Correspondence: yiguozhang@cqu.edu.cn or eaglezhang64@gmail.com

\section{Abstract}

In the past 25 years, Nrf2 had been preferentially parsed as a master hub of regulating antioxidant, detoxification and cytoprotective genes, albeit as a matter of fact that Nrf1, rather than Nrf2, is indispensable for cell homeostasis and organ integrity during normal growth and development. Here, distinct genotypic cell lines

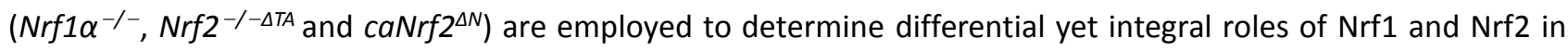
mediating antioxidant responsive genes to $t \mathrm{BHQ}$ as a pro-oxidative stressor. In $\mathrm{Nrf1 \alpha ^{-/- }}$ cells, Nrf2 was highly accumulated but also cannot fully compensate specific loss of Nrf1 $\alpha$ 's function in its basal cytoprotective response against endogenous oxidative stress, though it exerted partially inducible antioxidant response, as the hormetic effect of $t \mathrm{BHQ}$, against apoptotic damages. By contrast, $\mathrm{Nrf2} 2^{-/-\Delta \mathrm{TA}}$ cells gave rise to a substantial reduction of Nrf1 in both basal and $t \mathrm{BHQ}$-stimulated expression and hence resulted in obvious oxidative stress, but can still be allowed to mediate a potent antioxidant response, as accompanied by a significantly decreased ratio of GSSG to GSH. Conversely, a remarkable increase of Nrf1 expression was resulted from the constitutive active caNrf $2^{\Delta N}$ cells, which were not manifested with oxidative stress, no matter if it was intervened with $t B H Q$. Such inter-regulatory effects of Nrf1 and Nrf2 on antioxidant and detoxification genes (encoding HO-1, NQO1, GCLC, GCLM, GSR, GPX1, TALDO, MT1E and MT2), as well on the ROS-scavenging activities of SOD and CAT, were further investigated. The collective results unraveled that Nrf1 and Nrf2 make distinctive yet cooperative contributions to finely tuning basal constitutive and/or $t B H Q$-inducible expression levels of antioxidant cytoprotective genes in the inter-regulatory networks. Overall, Nrf1 acts as a brake control for Nrf2's functionality to be confined within a certain extent, whilst its transcription is regulated by Nrf2.

Keywords: Nrf1; Nrf2; antioxidant; oxidative stress; reactive oxygen species; tert-butylhydroquinone (tBHQ).

\section{Introduction}

With the development of science and technology, much more effective compounds with antioxidant properties have been discovered or synthesized insofar as to prevent lipid and protein oxidation [1, 2]. Amongst them, tert-butylhydroquinone ( $t B H Q)$ is a known small molecule phenolic antioxidant, which is a main metabolite of 3-tert-butyl-hydroxyanisole (BHA) in vivo in humans, dogs and rats, since it is widely used as a preservative in oils and processed foods $[3,4]$. However, $t \mathrm{BHQ}$ (and its precursor $\mathrm{BHA}$ ) is de facto identified as a double-faced compound with both effects to be exerted as an antioxidant and also a pro-oxidant in biological systems $[5,6]$. Of note, such a double-bladed sword impact of $t \mathrm{BHQ}$ is further unraveled by chemoprotective and carcinogenic effects of this compound and its reactive metabolites [6], in addition to its cytotoxicity [3, 4]. This is due to the fact that oxidative metabolism of $t \mathrm{BHQ}$ by metal-mediated redox cycling and microsomal monooxygenase system [e.g. phase I drug-metabolic enzyme cytochrome P450 1a1 (Cyp1a1)] yields several reactive oxygen species (ROS) and electrophilic intermediates, followed by the formation of reactive glutathione (GSH)-conjugates [e.g. in phase II drug-metabolic reactions by glutathione-S transferases (GSTs)]. Furtherly, $t$-BHQ is also identified to function as a novel ligand of aryl hydrocarbon receptor (AhR)[7], such that it can directly induce the expression of Cyp1a1, an enzyme known to play an important role in the chemical activation of xenobiotics to carcinogenic derivatives. Thereby, it is inferred that the AhR-dependent induction of Cyp1a1 by $t B H Q$ represents a positive feedback network so as to promote carcinogenicity, particularly upon its long-term exposure, in the gastrointestinal and liver tissues $[6,8]$.

On the another facet, the cytoprotective effect of $t \mathrm{BHQ}$ on biological systems is revealed by bona fide 
induction of the endogenous antioxidant and detoxification genes (e.g., those encoding phase II drug-metabolic enzymes) in response to this food additive[8, 9]. The endogenous antioxidant defense is provided predominantly by reduced glutathione (GSH) and other thiol-sensitive signaling molecules (e.g., thioredoxin), which contribute to metabolism of potentially harmful pro-oxidant agents (e.g., xenobiotics) and restore the intracellular redox balance to a steady-state, so that cell homeostasis is rebalanced $[5,10]$. Of note, the expression of such innate antioxidant biosynthetic and detoxifying enzymes is governed primarily by the Cap'n'Collar (CNC) basic region-leucine zipper (bZIP) family of transcription factors [11-13]. Amongst this family, Nrf1 and Nrf2 (both also called $\mathrm{Nfe} 2 \mathrm{l} 1$ and $\mathrm{Nfe} 2 \mathrm{l} 2$, respectively) are two principal regulators for maintaining robust redox homeostasis in life process [13-16]. To date, most studies of $t \mathrm{BHQ}$-induced antioxidant cytoprotective responses are focused disproportionately on the redox-sensitive $\operatorname{Nrf2}[8,11,17-19]$, rather than putative redox threshold-setting Nrf1 [20-22], since the former Nrf2 was firstly identified as a master regulator of the phase II detoxifying enzyme genes (e.g. NQO1, HO-1, GSTS) through their antioxidant response elements (AREs) to the pro-oxidant BHA or its metabolite $t \mathrm{BHQ}[5,23]$. The underlying mechanisms for $t \mathrm{BHQ}$-stimulated activity of Nrf2 are well documented $[11,24,25]$, but it is less understood whether and/or how the transactivation activity of Nrf1 is induced by exposure to this chemical.

Although Nrf2 is accepted as a master regulator of ARE-driven cytoprotective gene expression [11, 25], it is not essential for normal development and healthy growth, because its global knockout ( $\mathrm{Nrf2}^{-{ }^{-}}$) mice are manifested with neither any obvious defects nor spontaneous pathological phenotypes (e.g. cancer) [23, 26]. In effect, $\mathrm{Nrf2}{ }^{-/-}$mice are more susceptible than wild-type mice to chemical carcinogens [27], in addition to oxidative stress [28]. Thereafter, induction of $\mathrm{Nrf2}$ (by $t \mathrm{BHQ}$ ) has been recognized as a potential chemopreventive and therapeutic target against cancer $[25,29]$. To the contrary, the long-term induction of hyperactive Nrf2 is also reconsidered as a potent oncogenic driver with the hallmarks of cancer; this is based on its bona fide tumor-promoting effects and resistance to chemotherapy [30,31]. Such dual opposing roles of Nrf2 in cancer prevention and progression should be taken into account for its bidirectional potentials to implicate in cancer treatment.

By sharp contrast, Nrf1 is endowed with its innate unique features that are distinctive from Nrf2 [12, 32, 33], as evidenced by its gene-targeting knockout $\left(\mathrm{Nrf1}^{-/}\right)$to establish distinct animal models with significant pathological phenotypes [16, 20, 22, 34-36]. Global knockout of $\mathrm{Nrf1}^{-/-}$leads to embryonic lethality at E6.5 to E14.5, resulting from severe oxidative stress [20, 34, 35]. This fact implies that loss of Nrf1 cannot be compensated by Nrf2, though Nrf2 can also contribute to combinational regulation of antioxidant cytoprotective genes as confirmed by a double knockout model of $\mathrm{Nrf1}^{-/-}: \mathrm{Nrf2}^{-{ }^{--}}$[14]. Furtherly, distinct tissue-specific $\mathrm{Nrf1}^{-/-}$ mice are manifested with typical pathologies, resembling human non-alcoholic steatohepatitis (NASH) and hepatoma [16, 22], type-2 diabetes [37] and neurodegenerative diseases [38, 39]. Collectively, these demonstrate that mouse Nrf1 (and its isoforms) fulfills an indispensable function in regulating critical genes for maintaining robust redox homeostasis and organ integrity, so that the normal physiological development and growth are perpetuated in life process. However, it is regretable that these achievements are made mostly from mouse models. Such being the case, the underlying mechanism(s) by which human Nrf1 (or its derived isoforms) contributes to similar pathophysiological cytoprotective responses remains elusive.

For this reason, we have established three specific-knockout cell lines by gene-editing of human Nrf1 or Nrf2 on the base of HepG2 cells (named $N r f 1 \alpha^{-/-}, N r f 2^{-/-\Delta T A}$ and caNrf2 ${ }^{\Delta N}$, respectively) $[31,40]$. Herein, these three distinct genotypic cell lines together with wild-type cells were stimulated by $t \mathrm{BHQ}$ and subjected to a series of experimental interrogation of both basal and inducible expression levels of certain antioxidant, detoxification and cytoprotective genes. The resulting evidence has been presented by us, revealing that human Nrf1 and Nrf2 make differential, yet integral, contributions to synergistic regulation of antioxidant and detoxification genes induced by $t \mathrm{BHQ}$ as a pro-oxidative stressor. Of great note, it is plausible that the presence of Nrf1 determines basal redox steady-state and normal antioxidant cytoprotective responses against endogenous oxidative 
damages and apoptosis, albeit Nrf2 is involved in this homeostatic function. This study also provides a better understanding of the inter-regulatory roles of Nrf1 and Nrf2 within the redox control system, except that both factors can exert their specific yet combinational functions in the process. Such cautions should be severely taken into account for us to develop new drugs targeting either Nrf1 or Nrf2 alone or both in the future biomedical study.

\section{Materials and Methods}

\subsection{Cell lines and Regents}

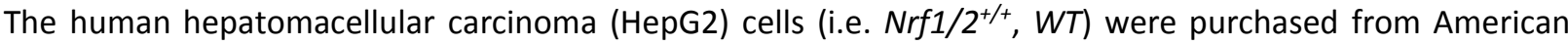
Type Culture Collection (ATCC, Manssas, VA, USA). And three HepG2-derived cell lines with distinct knockout types of $\mathrm{Nrf1}^{-/-}$(with a specific deletion mutant of full-length Nrf1/TCF11 and its derived isoforms), Nrf2 ${ }^{-/-\Delta T A}$ (lacking its longer transactivation domain-containing fragment), or caNrf2 ${ }^{\Delta N}$ (i.e. a constitutive active mutant of Nrf2 lacks its N-terminal Keap1-binding Neh2 domain) had been established in our laboratory, as described in detail by Qiu et al [31]. It is worth mentioning that the authenticity of HepG2 cell line had been confirmed by its authentication analysis and STR typing map (which was carried out by Shanghai Biowing Applied Biotechnology Co., Ltd., Shanghai, China). These cell lines were cultured in DMEM supplemented with $10 \%(v / v)$ FBS and 100 Units/L double-antibiotic (penicillin and streptomycin) and incubated at $37^{\circ} \mathrm{C}$ in a humidified $5 \% \mathrm{CO}_{2}$ atmosphere.

Subsequently, those experimental cells were treated with $t \mathrm{BHQ}$ (CAS No.1948-33-0, from Sigma), which is a newly synthesized phenolic antioxidant with the chemical formula of $\mathrm{C}_{10} \mathrm{H}_{14} \mathrm{O}_{2}$ at $\mathrm{MW} 166.22$. This compound has completely dissolved in DMSO at a final concentration of $50 \mu \mathrm{M}$ and stored at $-20^{\circ} \mathrm{C}$, before it is experimented. Of note, specific antibody against Nrf1 was made in our laboratory [41]. Besides, other five distinct antibodies against Nrf2 (ab62352), GCLC (ab207777), GCLM (ab126704), HO-1 (ab52947) or GPX1 (ab108427) were obtained from Abcam (Cambridge, UK). Additional antibodies against NQO1 (D26104), GSR (D220726) or TALDO1 (D623398) were from Sangon Biotech (Shanghai, China), whilst $\beta$-actin antibody (TA-09) was from ZSGB-BIO (Beijing, China).

\subsection{Cell viability with MTT assay}

All indicated experimental cells were digested by trypsin and diluted into a suspension of $5 \times 10^{4} \mathrm{cell} / \mathrm{mL}$, before being seeded into 96- well plates. After the cells were completely adherent to the plates, they were treated with $t B H Q$ at different concentrations (i.e. 0-100 $\mu \mathrm{M}$ ) for $24 \mathrm{~h}$, or with $50 \mu \mathrm{M}$ of $t \mathrm{BHQ}$ for distinct time periods (i.e. $0-24 \quad$ h). The cell viability was evaluated by assaying 3-(4,5-dimethylthiazol-2-yl)-2,5-diphenyltetrazolium bromide (MTT, ST1537, Beyotime, Shanghai, China) to form an insoluble product formazan in living cells. The resulting data were calculated by dividing the experimental absorbance by relevant control values. The results were shown as a percentage of mean $\pm S D(n=5)$, which are representative of at least three independent experiments.

\subsection{Quantitative RT-PCR analysis of mRNA expression}

Experimental cells growing in logarithmic phase were digested with trypsin and diluted by a complete medium into the suspension of $3.5 \times 10^{5} \mathrm{cell} / \mathrm{mL}$. Then equal amounts of cells were inoculated in 6 -well plates and cultured until being completely adherent. Thereafter, they were treated for different time periods (i.e. 0, 4, or $6 \mathrm{~h}$ ) with $50 \mu \mathrm{M}$ of $t \mathrm{BHQ}$. Subsequently, total RNA was isolated by using a RNA extrection kit (TIANGEN, Beijing,China), $500 \mathrm{ng}$ of which was then subjected to the reaction with reverse transcriptase (Promega, Madison, WI, USA) to synthesize the single strand CDNAs, that served as PCR templates. Basal and tBHQ-induced mRNA expression levels of those indicated genes were detected by quantitative real-time PCR (qRT-PCR) with each pairs of their primers (Table 1).

Table 1. The primer pairs used for qRT-PCR analysis 


\begin{tabular}{llll}
\hline ID & Name & Forward Primers (5'- 3') & Reverse Primers (5'-3') \\
\hline 60 & $\beta$-actin & CATGTACGTTGCTATCCAGGC & CTCCTTAATGTCACGCACGAT \\
$\mathbf{4 7 7 6}$ & Nrf1 & GAAGCCCACCAAGACCGAA & GCCTCTTCCTGTACACTGACC \\
$\mathbf{4 7 8 0}$ & Nrf2 & ATATTCCCGGCACATCGAGA & ATGTCCTGTTGCATACCGTCT \\
$\mathbf{2 7 2 9}$ & GCLC & TCAATGGGAAGGAAGTGTGTT & TCAATGGGAAGGAAGGTGTGT \\
$\mathbf{2 7 3 0}$ & GCLM & TCAATGGGAAGGAGGTGTGT & CGCTTGAATGTCAGGAATCTT \\
$\mathbf{2 8 7 6}$ & Gpx1 & CAGTCGGTGTATGCCTTCTCG & GAGGGACGCCACATTCTCG \\
$\mathbf{2 9 3 6}$ & GSR & CACGAGTGATCCCAAGCCC & CAATGTAACTGCACCAACAATG \\
6888 & TALDO & GGGCCGAGTATCCACAGAAG & GGCGAAGGAGAAGAGTAACG \\
$\mathbf{1 7 2 8}$ & NQO1 & AAGAAGAAGGATGGGAGGTGG & GAACAGACTCGGCAGGATACTG \\
$\mathbf{3 1 6 2}$ & HO-1 & CAGAGCCTGGAAGACACCCTAA & AAACCACCCCAACCCTGCTAT \\
$\mathbf{4 4 9 3}$ & MT1E & ATGGACCCCAACTGCTCTTGCGCCA & ACAGCAGCTGCACTTCTCCGATG \\
$\mathbf{4 5 0 2}$ & MT2 & GTGGGCTGTGCCAAGTGT & CAAACGGTCACGGTCAGG \\
\hline
\end{tabular}

The qRT-PCR reaction was carried out with GoTaq ${ }^{\circ} \mathrm{qPCR}$ Master Mix (Promega, Madison, WI, USA) on a CFX96 instrument (Bio-Rad, Hercules, CA, USA). The specific reaction procedure was followed by all samples, which were inactivated at $95{ }^{\circ} \mathrm{C}$ or $3 \mathrm{~min}$, and amplified by 40 reaction cycles of $15 \mathrm{~s}$ at $95^{\circ} \mathrm{C}$ and $30 \mathrm{~s}$ at $60{ }^{\circ} \mathrm{C}$. The resulting data were analyzed by the Bio-Rad CFX 96 Manager 3.0 software (Hercules, CA, USA), whilst $\beta$-actin expression level served an internal reference control and experimental values obtained from treatment with $t$-BHQ for $0 \mathrm{~h}$ (i.e. $T_{0}$ ) was used as relevant normal controls. The results of all examined genes were shown as fold changes (Mean $\pm S D, n=3$ ), which are representative of at least three independent experiments.

\subsection{Western blotting analysis of protein expression}

After experimental cells were collected as described above, the proteins were extracted for total cell lysates, diluted with a $3 \times$ loading buffer and denatured by boiling at $100^{\circ} \mathrm{C}$ for $10 \mathrm{~m}$. The samples from each of groups were electrophoretically separated by either $8 \%$ or $10 \%$ SDS-PAGE gels (running at $50 \vee$ for 30 min and then $100 \vee$ for $2 \mathrm{~h}$ ), before being transformed on the PVDF membrane (at $200 \mathrm{~A}$ for $2 \mathrm{~h}$ ). After the protein-botted membranes were blocked by $5 \%$ skimmed milk for $1 \mathrm{~h}$, they were incubated with each of the indicated primary antibodies at $4{ }^{\circ} \mathrm{C}$ overnight, and then re-incubated with the secondary antibody at room temperature for $2 \mathrm{~h}$. The protein blots were developed by the enhanced chemiluminescence as described previously [31]. The intensity of blots was calculated by using the Quantity One software (Bio-Rad Laboratories) and also normalized to $\beta$-actin as a loading control.

\subsection{Detection of cellular ROS and apoptosis by flow cytometry}

To determine intracellular ROS levels, all the cell samples were exposed to $100 \mu \mathrm{M}$ dichlofluorescein diacetate (DCFH-DA included in the detection kit, S0033S, Beyotime, Shanghai, China) for 30 min at the incubator $\left(37^{\circ} \mathrm{C}, 5 \% \mathrm{CO}_{2}\right)$. After being washed twice with PBS, the samples were centrifuged and resuspended in a serum-free medium. Then, the resulting 2'7'-dichlofluorescein was detected at the excitation wavelength of 488 $\mathrm{nm}$ and the emission wavelength of $525 \mathrm{~nm}$ by a flow cytometry (FlowJo, Ashland, OR, USA). In addition, the final results were expressed by the DCFH fluorescence intensity in distinct cells. Furthermore, those samples were collected by centrifuging at $1000 \times \mathrm{g}$ for $5 \mathrm{~min}$, and stained with binding buffer containing of Annexin V-FITC and propidium iodide (PI) for $15 \mathrm{~min}$. After the samples were washed twice to remove the excess staining reagent, they were subjected to detection of cell apoptosis by flow cytometry. The resulting data were shown by different fluorescence intensity in distinct states of cells.

\subsection{The assays for total, reduced and oxidized glutathione levels}

After experimental cells had been treated with $t \mathrm{BHQ}$ for indicated times (i.e. 0,4 or $6 \mathrm{~h}$ ), all cell samples were collected in PBS and then subjected to the measurement of total glutathione, reduced glutathione (GSH) and oxidized glutathione (GSSG) by using a Glutathione assay kit (A061-1, Nanjing Jiancheng, Nanjing, China) according to the manufacturer's instruction. Of note, two standards of GSH and GSSG were also prepared in the same assays. The assay was designed by using an Ellman's reagent ( 5,5 '- disulfidebis -2-nitrobenzoic acid, DNTB), 
which reacts with GSH to form 2- nitro-5- thiobenzoic acid, a yellow products with a absorbance at wavelength of $405 \mathrm{~nm}$. In addition, the protein concentrations in samples were determined by the bicinchoninic acid assay (BCA, P1511, ApplyGene, Beijing, China) and used as an internal control for the normalization, along with relevant standard curves, in order to calculate amounts of total glutathione, GSSG and GSH by the formula provided by this manufacturer. The final resulting data are shown by a ratio of GSSG to GSH levels.

\subsection{Assays for ROS-scavenging activities of superoxide dismutase and catalase.}

All groups of experimental cells had been treated with $t B H Q$ for distinct lengths of times $(0,4$ or $6 \mathrm{~h}$ ) before being collected and subjected to assays for ROS-scavenging activities of superoxide dismutase (SOD), that were determined according to the instruction of enhanced SOD assay kit (A001-3, Nanjing Jiancheng, China). The another ROS-scavenging enzyme catalase (CAT) activity was also detected through the instructions of CAT kit (BC0205, Solarbio, Beijing, China).

\subsection{ARE-Luciferase Reporter Assays}

All experimental cells were seed into 12 -well plates, after reaching $80 \%$ confluence, the cells were co-transfected using a lipofectamine 3000 mixture with each of $A R E$-driven luciferase plasmids (which were made by inserting each of the indicated ARE sequences into the pGL3-Promoter vector) or non-ARE reporter plasmids (as background control), together with an experiment construct for Nrf1, Nrf2 or empty pcDNA3.1 vector. In this test, the Renilla expression by pRL-TK plasmide served as an internal control for transfection efficiency. And the luciferase activity was measured by the dual-luciferase reporter system (Beyotime, Shanghai, China).

\subsection{Statistical analysis}

All the results are presented as mean $\pm S D$ ( $n=3$ or 5$)$. The comparison of the various experimental groups and their corresponding controls was carried out by one- way ANOVA, and analyzed by the post-hoc test with Fisher's least significant difference (LSD). The differences in between distinct treatments were considered to be statistically significant at $p<0.05$.

\section{Results}

\subsection{Different effects of Nrf1 and Nrf2 on cell growth during tBHQ intervention}

To gain an insight into endogenous Nrf1- and Nrf2-mediated antioxidant responses to $t \mathrm{BHQ}$, we here confirmed that four distinct genotypes of cell lines are true (Fig. 1A), as described previously [31, 40]. Of note, human Nrf1 $\alpha$ is manifested with four major isoforms, as identified by Xiang et al [41], of which its A and B isoforms represent the full-length glycoprotein and deglycoprotein of Nrf1, respectively, whilst its $C$ and $D$ isoforms denote two distinct lengths of Nrf1 N-terminally-truncated isoforms. Specific knockout of Nrf1 (by its gene-editing to delete a very short segment adjoining its translational start codons) led to a complete loss of all four $N r f 1 \alpha$-derived isoforms in $N r f 1 \alpha^{-/-}$cells, albeit with a retention of other two minor proteins $\mathrm{Nrf1} 1^{\Delta \mathrm{N}}$ and Nrf16 (Fig. 1A). By contrast, all four Nrf1 $\alpha$-derived isoforms A to D were also substantially diminished by $\mathrm{Nrf2} 2^{-/-\Delta T A}$, but its $B$ to $D$ isoforms (with distinct potentials of its trans-activity) were significantly augmented by caNrf2 $2^{\Delta N}$. Intriguingly, Nrf1B abundances were also markedly suppressed by $N r f 2^{-/-\Delta T A}$ or caNrf2 $2^{\Delta N}$. These imply that Nrf1 expression and processing may be monitored by Nrf2, besides itself. Conversely, only a major Nrf2 isoform-A, but not isoforms its B or C, was incremented in Nrf1 $\alpha^{-1-}$ cells (Fig. 1A). However, all three isoforms A to $\mathrm{C}$ of Nrf2 were completely abolished by specific deletion of its transactivation Neh4-Neh5 domains (to yield an inactive $N r f 2^{-/-\Delta T A}$, but their disappearance seemed to be replaced by another three smaller isoforms with a faster electrophoretic mobility when compared with equivalents examined in WT cells. By contrast, three slightly shorter isoforms of caNrf ${ }^{\Delta N}$ (closely to wild-type isoforms A to C, respectively) were retained and enhanced by this constitutive active mutant factor, because the N-terminal Keap1-binding Neh2 domain of Nrf2 was removed from its genomic locus. Collectively, these indicate that Nrf2 expression and processing may also be monitored by itself, as well by Nrf1, within an inter-regulatory feedback cycle. 
Next, the cytotoxic effect of $t \mathrm{BHQ}$ on aforementioned four different cell lines was evaluated by a MTT assay for the formation of formazan precipitates with succinate dehydrogenase in the mitochondria of all living cells only, and changes in the absorbance were measured to reflect the cell viability. As shown in Fig.1B, the viability of all four examined cell lines was modestly decreased by intervention with $5 \mu \mathrm{M} t \mathrm{BHQ}$, but $10 \mu \mathrm{M}$ of this chemical enabled these cell viability to return closely to their basal levels. Then, a relatively stable viability of $\mathrm{Nrf1} 1 \alpha^{-/-}$cells was maintained between $10-60 \mu \mathrm{M}$ of $t \mathrm{BHQ}$, followed by a gradual decrease to $80 \%$ of its viability until its concentration increased to $100 \mu \mathrm{M}$ (Fig. 1B). By contrast, a narrow window of stable WT cell viability was defined by $10-20 \mu \mathrm{M} t \mathrm{BHQ}$, followed by a fairly sloping downhill to $70 \%$ viability of the cells treated with $100 \mu \mathrm{M}$ $t \mathrm{BHQ}$, while other two close smoothly growth curves emerged from 10 to $80 \mu \mathrm{M} t \mathrm{BHQ}$ treatments of both $\mathrm{Nrf2} 2^{-/-\Delta T A}$ and caNrf2 ${ }^{\Delta N}$ cell lines, before their viability decreased to $80 \%$ and $70 \%$, respectively, upon treatment of $100 \mu \mathrm{M} t \mathrm{BHQ}$ (Fig. 1B).

Based on the dose-dependent effects, $50 \mu \mathrm{M} t \mathrm{BHQ}$ was selected for intervention of the above-described four cell lines to assess distinct time-dependent growth courses (Fig. 1C). The results showed that the viability of all four cell lines decreased to different extents of between $90 \%$ and $75 \%$ by $t$ BHQ intervention for $1 \mathrm{~h}$. Of note, the continuous treatment enabled the viability of $W T$ and $N r f 2^{-/-\Delta T A}$ cell lines to smoothly decrease to $85 \%-80 \%$ or $75 \%-75 \%$ from $2 \mathrm{~h}$ or $4 \mathrm{~h}$ to $24 \mathrm{~h}$, respectively (Fig. 1C). By sharp contrast, the viability of $\mathrm{Nrfl}^{-\alpha^{-/}}$and caNrf2 ${ }^{\Delta \mathrm{N}}$ cell lines appeared to elevate respectively to $100 \%$ or $90 \%$ in a modest 'bounce-back' response to $t \mathrm{BHQ}$-continued treatment from $2 \mathrm{~h}$ to $4 \mathrm{~h}$, and then both declined to $75 \%$ at $12 \mathrm{~h}$ of treatment. Thereafter, the viability of $\mathrm{Nrf1}^{-/-}$cells continued to gradually reduce to $70 \%$ until $24 \mathrm{~h}$ of $t \mathrm{BHQ}$ treatment, whilst the viability of caNrf2 $2^{\Delta N}$ cells was maintained to $75 \%$ from $12 \mathrm{~h}$ to $24 \mathrm{~h}$ treatments. As such, all cell viability reached a relatively stable level of them after $16 \mathrm{~h}$ of $t$-BHQ intervention. Therefore, the optimal concentration of $t \mathrm{BHQ}$ and its optimal time course were selected in the follow-up experiments to assess the cytoprotective roles of Nrf1 and Nrf2 against this chemical. For this end, we mainly investigated their expression differences between these four cell lines in responses to $50 \mu \mathrm{M} t$-BHQ intervention for $0,1,2,4$ and/or $16 \mathrm{~h}$.

\subsection{Short-term intervening effects of tBHQ on Nrf1, Nrf2 and AREs-driven genes in distinct genotypic cells}

Herein, short-term effects of $t \mathrm{BHQ}$ intervention for 1-2 h on Nrf1 and Nrf2 were examined by western blotting (Fig. 1, D \& E). The results showed that $t \mathrm{BHQ}$ treatment of $W T$ cells caused modest increases in Nrf1-processed isoforms C/D, as well as $\mathrm{Nrf1}^{\Delta N}$ (Fig. 1D, d1). Such altered $N r f 1^{\Delta N}$ also emerged in $N r f 1 \alpha^{-/-}$cells, albeit it lacked $A$ to $D$ isoforms, implying it is not originated from the full-length $N r f 1 \alpha$ processing. By contrast, a slight enhancement in the remnant $\mathrm{Nrf1} \alpha$-derived isoforms and $N r f 1^{\Delta N}$ expression in $t \mathrm{BHQ}$-treated $\mathrm{Nrf2} 2^{-1-\Delta T A}$ cells, but both their basal and $t \mathrm{BHQ}$-stimulated levels were increased in caNrf2 $2^{\Delta N}$ cells (Fig. 1D, d1). For Nrf2, its protein expression was more sensitive to $t B H Q$ stimulation in $W T$ cells, and also increased significantly after $1 \mathrm{~h}$ treatment (Fig. 1D, d2), when compared with those in the other three cell lines, which appeared to be largely insensitive to $t \mathrm{BHQ}$, even although altered $\mathrm{Nrf2}$ expression levels were evidently enhanced in $\mathrm{Nrf1 \alpha ^{-/- }}$ and $c a N r f 2^{\Delta N}$, except $N r f 2^{-1-\Delta T A}$, cells lines, but with no obvious changes after $t B H Q$.

Both basal and tBHQ-stimulated expression levels of ARE-driven genes regulated by Nrf1 and/or Nrf2 were determined, next (Fig. 1, D \& E). The results revealed that distinct expression levels of NQO1 (NAD(P)H:quinone oxidoreductase 1; Fig.1D, d4), GCLM (glutamate-cysteine ligase modifier subunit; Fig.1E, e2), GPX1 (glutathione peroxidase 1; Fig.1E, e4) and HO-1 (heme oxygenase 1, also called HMOX1; Fig.1E, d4) in WT cells were induced by $t B H Q$; this appeared to be accompanied by Nrf2 inducible enhancement. However, all these examined proteins, also including GCLC (glutamate-cysteine ligase catalytic subunit; Fig.1E, e1), GSR (glutathione-disulfide reductase, e3) and TALDO (transaldolase $1, e 5$ ), were largely unaffected by short-term tBHQ intervention of $\mathrm{Nrf1}^{-/-}$cells, even though basal abundances of NQO1, GCLM and GPX1, amongst them aforementioned, were highly augmented as accompanied by hyper-expression of Nrf2. Similarly, constitutive active caNrf $2^{\Delta N}$ also resulted in basal increases in GCLM, GPX1 and TALDO (Fig. 1E, e2, e4, e5) as accompanied by a basal decrease of NQO1, but all these examined protein levels were almost unaltered by $t \mathrm{BHQ}$ stimulation of caNrf ${ }^{\Delta N}$ cells. 
Conversely, knockout of $\mathrm{Nrf2}^{-/-\Delta T A}$ only led to reduced basal levels of both HO-1 and GSR (Fig.1D, d3 \& Fig. 1E, e3),

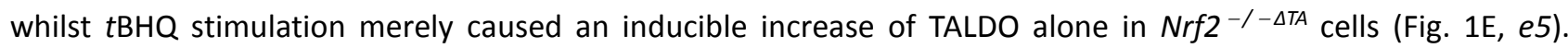
Intriguingly, $t \mathrm{BHQ}$-triggered $\mathrm{Nrf2^{-/- \triangle TA }}$ cells also gave rise to modest decreases of NQO1, GCLM, or GPX1 (Fig.1D, $d 4$ \& Fig. 1E, e2, e4). Altogether, these indicate that Nrf1 and Nrf2 could make differential yet integral contributions to basal and $\mathrm{BHO}_{\mathrm{B}}$-inducible expression levels of these examined ARE-driven genes. For further insights into differential expression patterns of these antioxidant cytoprotective genes among different genotypic cell lines, the following experiments were performed by long-term stimulation of cells with $t B H Q$ for $4 h$ to $24 \mathrm{~h}$.

\subsection{Long-term stimulating effects of tBHQ on Nrf1, Nrf2 and downstream targets in distinct genotypic cells}

To give a proper understanding of long-term tBHQ-stimulated effects on Nrf1, Nrf2 and downstream genes, their mRNA expression levels were determined by quantitative real-time PCR (Fig. 2). The results revealed an obvious increase of $N r f 1$ mRNA expression after 12-h tBHQ stimulation of WT cells (Fig. 2A). By contrast, basal

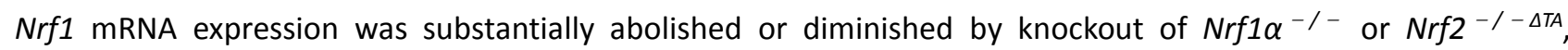
respectively. Therefore, although $\mathrm{Nrf2}$ was highly expressed in $\mathrm{Nrf1}^{-\alpha^{-1}}$ cells (Figs. 1D \& 2B), its remnant $\mathrm{Nrf1}$ was

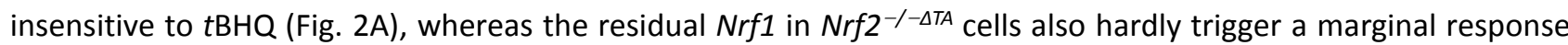
to this chemical. Conversely, caNrf2 $2^{\Delta N}$ cells had given rise to a remarkable increase in basal Nrf1 mRNA levels, but only a modest $t \mathrm{BHQ}$-inducible increase of $\mathrm{Nrf1}$ expression was detected after 20-24 $\mathrm{h}$ stimulation of this cell lines (Fig. 2A). These results indicate that transcriptional expression of human Nrf1 gene is monitored by Nrf2, as well by Nrf1 itself.

Treatment of $W T$ cells with $t \mathrm{BHQ}$ caused a gradual modest induction of Nrf2 mRNA expression levels from $8 \mathrm{~h}$ to $16 \mathrm{~h}$, followed by a marked peak of its induction at $24 \mathrm{~h}$, of this chemical stimulation (Fig. 2B). Both basal and $t$ BHQ-stimulated $\mathrm{Nrf2}$ expression levels were abolished by $\mathrm{Nrf2}^{-1-\Delta T A}$. Interestingly, even though basal $\mathrm{Nrf2} \mathrm{mRNA}$ expression was significantly augmented by $\mathrm{Nrf1}^{-/-}$or caNrf2 $2^{\Delta N}$, its $t \mathrm{BHQ}$-stimulated expression was unaffected or partially reduced in such two distinct genotypic cell lines (Fig. 2B). Collectively, these indicate that transcriptional expression of human Nrf2 gene is bidirectionally regulated by itself and Nrf1, but upon stimulation by $t \mathrm{BHQ}$, itself regulation by Nrf2 per se appears to be attributable to its $\mathrm{N}$-terminal Keap1-binding Neh2 domain.

Besides Nrf1 and Nrf2, downstream target genes $\mathrm{HO}-1$ and $\mathrm{NQO} 1$ were also induced by $t \mathrm{BHQ}$ of $W T$ cells in a time-dependent manner (Fig. 2, C \& D). Upon loss of Nrf1 $\alpha$-derived isoforms, significant increments in basal and $t \mathrm{BHQ}$-stimulated mRNA expression levels of $\mathrm{HO}-1$ and $\mathrm{NQO} 1$ were determined in $\mathrm{Nrfl}^{-/-}$cells. The first sharp maximum peak of $\mathrm{HO}-1$ occurred at $4 \mathrm{~h}$ of induction by $t \mathrm{BHQ}$, followed by a gradual decline to $16 \mathrm{~h}$ and then the second peak at $20 \mathrm{~h}$ treatment of $\mathrm{Nrf1}^{-/-}$cells (Fig. 2C). By contrast, only a smooth increase of NQO1 was obtained from $4 \mathrm{~h}$ to $12 \mathrm{~h}$ of $t \mathrm{BHQ}$ induction of $\mathrm{Nrf} 1 \alpha^{-/-}$cells to a higher level, which was then maintained at such high level until $24 \mathrm{~h}$ (Fig. 2D). However, loss of $\mathrm{Nrf2}^{-/-\Delta \mathrm{TA}}$ led to an evident diminishment or abolishment in basal and $t \mathrm{BHQ}$-stimulated expression levels of $\mathrm{HO}-1$ and $\mathrm{NQO1}$ (Fig. 2, C \&D), except for a marginal induction of $N Q O 1$ by $t \mathrm{BHQ}$ at $24 \mathrm{~h}$. Conversely, constitutive active caNrf2 $2^{\triangle N}$ appeared to have no significant effects on both basal and $t$ BHQ-stimulated expression of HO-1 and NQO1 (Fig. 2, C \&D), albeit with a weak induction of NQO1 by $t \mathrm{BHQ}$ at $24 \mathrm{~h}$. Altogether, these data indicate that $\mathrm{HO}-1$ and NQO1 serve as two representative targets of Nrf2, and both gene regulation by Nrf2 may also be monitored positively by its N-terminal Neh2 domain, aside from the potential negative regulation of Nrf2 and its targets by Nrf1.

\subsection{Long-term stimulation of human antioxidant and detoxification genes by $t B H Q$ in distinct genotypic cells}

It is of crucial antioxidant and detoxification to be merited by glutathione (GSH)-conjugates in redox signaling cycles. The intracellular biosynthesis of GSH, as an important cellular antioxidant, is controlled by a key rate-limiting enzyme consisting of both GCLC and GCLM subunits. As shown in Fig. 2 (E \& F), a time-dependent increment in the mRNA expression of GCLC and GCLM induced by $t B H Q$ from $4 \mathrm{~h}$ to $24 \mathrm{~h}$ was determined in $W T$ cells. By contrast, $\mathrm{Nrfl}^{-/-}$cells gave rise to a rapid induction of GCLC mRNA expression by 4-h of $t B H Q$ 
stimulation, to a maximum peak similar to that of $W T$ cells, which was then maintained at such higher level until $24 \mathrm{~h}$ (Fig. 2E). However, no striking changes in both basal and tBHQ-stimulated GCLC expression were observed in $N r 2^{-/-\triangle T A}$ or caNrf2 $2^{\Delta N}$ cell lines. Interestingly, further examinations revealed that basal and $t \mathrm{BHQ}$-stimulated GCLM expression levels were substantially augmented in $\mathrm{Nrf1}^{-/-}$cells (Fig. 2F), whilst caNrf2 ${ }^{\Delta N}$ cells only gave rise to a relatively lower induction of GCLM by $t B H Q$, even though its basal expression was elevated at a similar level to that obtained from $\mathrm{Nrf1} \alpha^{-/-}$cells. In addition, $\mathrm{Nrf2} 2^{-/-\Delta T A}$ cells still retained a considerable lower induction of GCLM by $t$ BHQ (Fig. 2F). Taken together, these results demonstrate differential contributions of Nrf1 and Nrf2 to basal and inducible regulation of both GCLC and GCLM genes controlling GSH biosynthesis in distinct genotypic cells.

As a central enzyme of the intracellular antioxidant defense, GSR can reduce the oxidized glutathione disulfide (GSSG) to the sulfhydryl form (GSH). In such a thiol-based redox cycle, another key enzyme GPX1, belonging to the glutathione peroxidase family, can catalyze the glutathione to reduce hydrogen peroxide $\left(\mathrm{H}_{2} \mathrm{O}_{2}\right)$ and other organic hydroperoxides, in order to detoxify the oxidants and hence protect the cells from oxidative damages. Thereby, we examine the intervening effects of $t B H Q$ on induction of GSR and GPX1 mRNA expression mediated by Nrf1 and/or Nrf2 in distinct genotypic cell lines. As anticipated, RT-qPCR results revealed that GSR mRNA levels were strikingly gradually up-regulated by $t \mathrm{BHQ}$ stimulation of $W T$ cells from $8 \mathrm{~h}$ to $24 \mathrm{~h}$ (Fig. 2G),

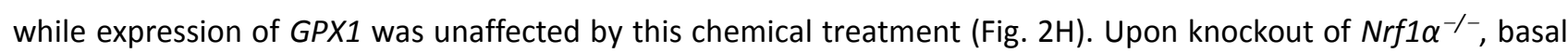
mRNA levels of GSR and GPX1 were markedly enhanced, but only modest induction of GSR, rather than GPX1, by $t \mathrm{BHQ}$ occurred from $4 \mathrm{~h}$ to $8 \mathrm{~h}$ and thereafter maintained at a maximum level that was lower than equivalent values measured from $t \mathrm{BHQ}$-treated $W T$ cells (Fig. 2,G \& H). Such $t \mathrm{BHQ}$-trigged induction of $G S R$, as well as its basal expression levels, was substantially attenuated or abolished by knockout of $\mathrm{Nrf2}^{-/-\Delta T A}$ (Fig. 2G). However,

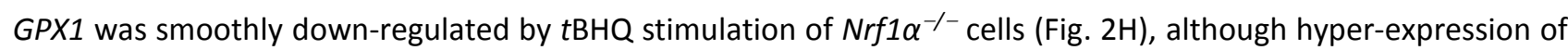
Nrf2 was preserved in this knockout cell line (Fig. 2B), but this effect appeared to be completely prevented by $N r 2^{-/-\Delta T A}$ (Fig. 2H). Conversely, constitutive active caNrf2 ${ }^{\triangle N}$ only gave rise to a remarkable increase in basal expression levels of GPX1, but not GSR, except that both genes were almost insensitive to $t$ BHQ stimulation (Fig. 2, G \& H).

Furthermore, TALDO is a key enzyme of the non-oxidative pentose phosphate pathway (PPP) providing ribose-5-phosphate for nucleic acid synthesis and NADPH for lipid biosynthesis [42]. Notably, NADPH arising from this pathway enables glutathione to be maintained at a reduced state, thereby protecting sulfhydryl groups and cellular integrity from oxygen radicals. Herein, our results unraveled a stepwise inducible increase of TALDO mRNA expression levels from $4 \mathrm{~h}$ to $20 \mathrm{~h}$ of its maximum stimulation by $t B H Q$ of $W T$ cells, before being maintained until $24 \mathrm{~h}$ of this chemical stimulation (Fig. 2I). By contrast, $\mathrm{Nrf1} \alpha^{-/-}$caused significant increments in basal and $t B H Q$-stimulated expression levels of TALDO from $4 \mathrm{~h}$ to $12 \mathrm{~h}$ of its rapidly inducible peak that was much higher than the values from WT control cells, which was largely retained to $24 \mathrm{~h}$. Such induction of TALDO was almost abolished by $\mathrm{Nrf2}^{-/-\Delta T A}$ (except for a marginal induction of it by 24-h stimulation of $t \mathrm{BHQ}$ ), and also suppressed by caNrf2 $2^{\Delta N}$ (albeit its basal levels were augmented) (Fig. 2I). Collectively, these indicate that Nrf1 and Nrf2 exert differential yet integral roles in mediating the aforementioned antioxidant cytoprotective genes against $t B H Q$.

In addition, it is worth mentioning that metallothioneins (MT) cannot only maintain the metal homeostasis in vivo, but also serve as a redox buffer for ROS and other free radicals to play an essential role in the cytoprotection [43]. However, our examinations of MT1E and MT2 unraveled that both genes were not merely insensitive to $t \mathrm{BHQ}$, but were modestly down-regulated by this chemical intervention of $W T$ cells (Fig. 2, J \& $\mathrm{K}$ ). Of note, basal mRNA expression of MT1E, along with its inhibitory effect of $t B H Q$ were markedly diminished or completely abolished in caNrf2 $2^{\Delta N}$ or $N r f 1 \alpha^{-/-}$cells, respectively (Fig. 2J), but both cell lines gave rise to a remarkable increase of basal MT2 expression, aside from that $t \mathrm{BHQ}$-stimulated expression of $M T 2$ was elevated in caNrf2 $2^{\Delta N}$ rather than $N r f 1 \alpha^{-/-}$cells (Fig. $2 \mathrm{~K}$ ). By contrast, $N r f 2^{-/-\Delta T A}$ cells gave rise to significant increases in 
basal and $t B H Q$-inducible mRNA expression profiles of $M T 1 E$ from $4 \mathrm{~h}$ to $12 \mathrm{~h}$ of this chemical stimulation, prior to being maintained at a considerable higher levels until $24 \mathrm{~h}$ (Fig. 2J), whereas basal MT2 expression level was

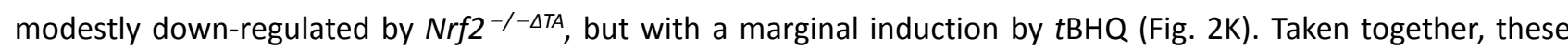
suggest distinct contributions of Nrf1 and Nrf2 to transcriptional regulation of MT1E and MT2, respectively.

\subsection{Distinct time-dependent effects of $t B H Q$ on Nrf1, Nrf2 and target gene expression in different cell lines}

As shown in Fig. 3 (A, a1), Nrf1 $\alpha$-derived isoforms A to D were obviously enhanced after $4 \mathrm{~h}$ of $t \mathrm{BHQ}$ treatment in $W T$ cells and then maintained to their considerably higher extents between $8 \mathrm{~h}$ and $24 \mathrm{~h}$, as illustrated graphically (Fig. 3A, a6). Similarly, the abundance of Nrf2 proteins was rapidly significantly augmented by $t \mathrm{BHQ}$ stimulation of $W T$ cells from $1 \mathrm{~h}$ to $24 \mathrm{~h}$ (Figs. 1D, d2 \& 3A, a2). Although hyper-expressed Nrf2 was retained in $\mathrm{Nrf1} \alpha^{-/-}$cells, its protein abundances were unaffected by $t \mathrm{BHQ}$ intervention of this Nrf1 $\alpha$-specific knockout cells (Fig, 2B, b2, b6), in which Nrf1 $\alpha$-derived isoforms were constitutively lacked, but the $\mathrm{N}$-terminal portion-truncated Nrf1 ${ }^{\Delta N}$ abundances were promoted by $t \mathrm{BHQ}$ (Fig, 2B, b1). By contrast, Nrf2 ${ }^{-/-\Delta T A}$ cells only yielded considerably weaker abundances of Nrf1 $\alpha$-derived isoforms $A$ to $D$, but they still enabled to respond $t \mathrm{BHQ}$ in a biphasic manner, with the first peak at $4 \mathrm{~h}$ of this stimulation and the recurring second peak at $20 \mathrm{~h}$ (Fig. $2 \mathrm{C}, c 1 \& c 6$ ), whilst the remnant $\mathrm{Nrf2}^{\triangle \mathrm{TAD}}$ protein was evidently time-dependently inhibited by $t \mathrm{BHO}$ intervention of $\mathrm{Nrf2}^{-/-\Delta \mathrm{TA}}$ cells (Fig. $2 \mathrm{C}, \mathrm{c2}$ ). Conversely, caNrf2 ${ }^{\Delta N}$ cells led to strikingly increased abundances of basal $\mathrm{Nrf}^{\Delta \mathrm{N}}$ and Nrf1 $\alpha$-derived isoforms (Fig. 1D, $d 2 \& d 1$ ), and also their time-dependent inducible expression changes were herein determined in $t \mathrm{BHQ}$-stimulated caNrf2 ${ }^{\triangle N}$ cells (Fig. 3D, $d 1, d 2 \& d 6$ ).

Next, further examinations revealed that $t \mathrm{BHQ}$-inducible expression levels of $\mathrm{HO}-1$ in WT cells were gradually incremented, as its intervening time extended from $4 \mathrm{~h}$ to $20 \mathrm{~h}$, to a considerably higher level, before being slightly declined (Fig. 3A, a3 \& a6), whilst NQ01-induced expression levels were rapidly triggered by 4-h of this stimulation to a certain extent, and then maintained until $24 \mathrm{~h}$ (Fig. 3A, a4 \& a6). Of great note, although basal abundance of HO-1 was substantially diminished by $\mathrm{Nrf2}^{-/-\Delta T A}$ (Fig. 1D, d3), it remained to be significantly induced by $t \mathrm{BHQ}$ from $8 \mathrm{~h}$ to $24 \mathrm{~h}$ of stimulation to a maximum extent (Fig. 3C, $c 3$ \& C6). In Nrf1 $1 \alpha^{-/-}$cells, even though the hyper-expressed Nrf2 was insensitive to $t \mathrm{BHQ}$, both $\mathrm{HO}-1$ and NOQ1 were still rapidly induced by this chemical from $4 \mathrm{~h}$ to $8 \mathrm{~h}$ of stimulation and then maintained to their respectively higher extents until $24 \mathrm{~h}$ (Fig, $3 \mathrm{~B}, b 2-b 4, b 6)$. Rather, only a marginal induction of $c a N r f 2^{\Delta N}$ by $t \mathrm{BHQ}$ was observed, but this was accompanied by significant induction of HO-1 and NQO1, as well as Nrf1 $\alpha$-derived proteins, in their distinct time-dependent courses (Fig. 3D, d1-d6). Altogether these collective results imply that such two inter-regulatory factors of Nrf1 and Nrf2 could mediate differential yet integral responses to $t \mathrm{BHQ}$ intervention of different genotypic cell lines.

\subsection{Different time-dependent effects of $t B H Q$ on antioxidant cytoprotective gene expression in distinct cell lines}

As shown in Fig. 4A, tBHQ stimulation of $W T$ cells caused significant time-dependent induction of GCLC, GCLM, GSR, GPX1, and TALDO ( $a 1$ to $a 7$ ). Amongst them, GCLC was relatively slowly induced after $8 \mathrm{~h}$ of $t \mathrm{BHO}$ stimulation and then gradually incremented to a maximum inducible extent at $20 \mathrm{~h}$ of this chemical treatment, before being slightly declined $(a 1, a 7)$. By contrast, GCLM, GSR and TALDO was rapidly induced within $4 \mathrm{~h}$ of stimulation by $t \mathrm{BHQ}$ and then presented in stepwise ascend from $8 \mathrm{~h}$ to $24 \mathrm{~h}$ of this treatment $(a 2, a 3, a 5 \& a 7)$, whilst GPX1 induction by $t \mathrm{BHQ}$ appeared to rise and fall in wave modes $(a 4, a 7)$. Such differences in these examined enzymes may be attributable to distinct involvement of their upstream factors (e.g., Nrf1 and Nrf2) in response to $t B H Q$.

Next, we determine distinct contributions of Nrf1 and Nrf2 to such alterations in tBHQ-stimulated abundances of antioxidant and detoxification enzymes. As revealed in $\mathrm{Nrf1}^{-/-}$cells, GCLC and GCLM were successively induced by $t \mathrm{BHO}$ from $4 \mathrm{~h}$ to $24 \mathrm{~h}$ of their maximum stimulation (Fig. $4 \mathrm{~B}, b 1, b 2 \& b 7$ ), while a lag induction of GSR occurred at $8 \mathrm{~h}$ of $t \mathrm{BHQ}$ stimulation, which was maintained to $16 \mathrm{~h}$ and then declined gradually to its basal levels $(b 3, \& b 7)$. As such, TALDO only displayed modest induction by $t$ BHQ in a biphasic stepwise, 
with the first induction at $8 \mathrm{~h}$ and the second higher induction at $20 \mathrm{~h}$ before be declined nearly to its basal level ( $b 5 \& b 7$ ), except largely no induction of GPX1 in $\mathrm{Nrf1}^{-/-}$cells $(b 4 \& b 7)$, although Nrf2 was hyper-expressed. However, Nrf2 $2^{-1-\Delta T A}$ cells still gave rise to gradually-increased abundances of GCLC, GCLM, GPX1 and TALDO from $4 \mathrm{~h}$ to $24 \mathrm{~h}$ of $t \mathrm{BHQ}$ stimulation (Fig. 4C, $c 1, c 2 \&(4-c 7)$ ), whereas GSR was slightly down-regulated by this chemical ( $c 3 \& c 7$ ). By contrast, caNrf2 ${ }^{\Delta N}$ only led to a significant increment in induction of GCLM by $t$ BHQ from 4 $\mathrm{h}$ to $24 \mathrm{~h}$ (Fig. 4D, $d 2 \& d 7$ ), in addition to modest induction of GCLC, GPX1, TALDO, but not GSR, by this stimulation, which was maintained from $8 \mathrm{~h}$ to $12 \mathrm{~h}$ and then declined to relatively lower levels $(d 1, d 3-d 7)$.

\subsection{Different antioxidant responses of four distinct genotypic cell line to tBHQ as a pro-oxidative stressor}

The above experiments revealed there exists a synergistic effect of those antioxidant and detoxification genes regulated by Nrf1 and/or Nrf2. Just such synergistic effects can fully ensure the stable and effective function of this antioxidant cytoprotective system (to yield GSH and NADPH) to remove the excessive ROS produced from oxidative stressor, so that a certain redox homeostasis is being maintained to ensure the proper physiological operation of a heathy body. As a general term, ROS represents a set of all oxygen-containing reactive substances, including superoxide anion, hydrogen peroxide and relevant free radicals. To date, they remain to be hardly detected, owing to their characteristics of strong oxidative activity with such a short life to be rapidly scavenged and detoxified by antioxidants (i.e., GSH). As such, the intracellular redox state was herein measured directly by DCFH-DA, one of the most widely-used assays to evaluate the resulting oxidative damages, because it can react directly with ROS to give rise to an extremely sensitive, but impermeable, dichlofluorescein probe, as detected by flow cytometry [44]. As shown in Fig. 5A, a left shift of the dichlofluorescein image resulted from 16-h tBHQ intervention of $W T$ cells (a1, also see Fig. S1), implying a relative decrease of intracellular ROS levels, when compared with the control image obtained from the vehicle treatment (at $0 \mathrm{~h}$ ). Further examinations revealed that $\mathrm{Nrf1}^{-/-}$or $\mathrm{Nrf2} 2^{-/-\Delta \mathrm{TA}}$ gave rise to a significant increase in basal ROS levels under the vehicle-treated conditions, and also an evident left-shift of their $t \mathrm{BHQ}$-intervening images to varying extents (Fig. 5A, a2, a3 \& 5B, and Fig. S1). This indicates that loss of stimulated by $t \mathrm{BHQ}$ to trigger antioxidant cytoprotective responses against the endogenic ROS. Intriguingly, almost no changes in both basal and $t B H Q$-stimulated dichlofluorescein images were determined in caNrf2 ${ }^{\Delta N}$ cells, when compared to $W T$ cells (Figs. 5A, a3 \& 5B, and Fig. S1), implying that the N-terminal Keap1-binding domain of Nrf2 is required for $t \mathrm{BHQ}$-triggered antioxidant response.

Further glutathione assays unraveled that the ratio of GSSG to GSH was marginally reduced by $t B H Q$ stimulation of WT cells (Fig. 5C). By sharp contrast, $\mathrm{Nrf1}^{-/ \alpha^{--}}$led to a remarkable increase in its basal GSSG to GSH ratio, but significant decreases of this ratio occurred after $t \mathrm{BHQ}$ stimulation. This indicates putative endogenous oxidative stress to yield the excessive GSSG, much more than GSH levels, in this Nrf2-hyperexpressed $\mathrm{Nrf1}^{-/-}$cells, but the remaining antioxidant response in this cell line may be still triggered by $t B H Q$. Contrarily, $N r f 2^{-1-\Delta T A}$ and $c a N r f 2^{\triangle N}$ further caused substantial decreases in their basal GSSG to GSH ratio, although their stimulated ratios were less or not promoted by $t \mathrm{BHQ}$, respectively (Fig. $5 \mathrm{C}$ ). This implicates such two distinctive mutants enable to yield a certain amount of GSH in $N r f 2^{-/-\Delta T A}$ and caNrf2 $2^{\Delta N}$ cell lines, but could not allow for effective conversion of GSH into GSSG, even under $t$ BHQ-stimulated conditions.

To gain insights into the initial scavengers of ROS, the activity of two key enzymes, superoxide dismutase (SOD) and catalase (CAT) was examined herein. As shown in Fig. 5D, significant increases in the basal activity of SOD were determined in $\mathrm{Nrf1}^{-/-}, \mathrm{Nrf2} 2^{-/-\Delta T A}$ or caNrf2 $2^{\Delta N}$ cell lines, and tBHQ-stimulated activity of SOD was further promoted only in $N r f 1 \alpha^{-/-}, N r f 2^{-/-\Delta T A}$, but not caNrf2 $2^{\Delta N}$, cell lines. Of note, a longer term (16 h) treatment of $\mathrm{Nrf2}^{-/-\Delta T A}$ cells with $t B H Q$ caused a substantial reduction of SOD activity to its basal level, which was, though, still higher than that measured from $W T$ cells (Fig. 5D). However, caNrf2 ${ }^{\Delta N}$ cells displayed no significant changes in $t$ BHQ-inducible SOD activity, albeit its basal activity was much more than that of $W T$ cells. Also, no obvious changes in the SOD activity were detected in $W T$ cells that had or had not been treated by $t B H Q$. However, further examinations revealed that CAT activity was evidently stimulated by $t B H Q$ in $W T$ cells (Fig. 5E). By 
contrast, basal CAT activity was increased in both cell lines of $\mathrm{Nrfl}_{1} \alpha^{-/-}$and $\mathrm{Nrf2} 2^{-/-\Delta \mathrm{TA}}$, but its $t \mathrm{BHQ}$-stimulated

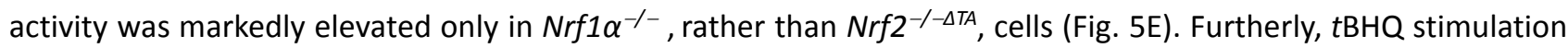
of caNrf2 ${ }^{\Delta N}$ cells caused a striking suppression or even complete abolishment of its inducible CAT activity at $4 \mathrm{~h}$ or $16 \mathrm{~h}$ of this treatment, respectively, although its basal activity was greatly substantially augmented. Such discrepant activities of SOD and CAT in between these cell lines, together with their differential expression results published previously [45], demonstrate to be attributable to distinctive yet cooperative contributions of Nrf1 and Nrf2 at regulating different target genes.

\subsection{Distinct roles of Nrf1 and Nrf2 in different cell apoptosis induced by tBHQ as a pro-oxidative stressor}

Further analysis by flow cytometry unraveled that only a few number of apoptotic cells were indeed examined in $W T$ cells that had been intervened with $t$ BHQ for $16 \mathrm{~h}$ (Fig. 6, A \& B). By contrast, a remarkable augment in basal apoptosis of $\mathrm{Nrfl}^{-/-}$cells reached to a much higher rate than that of the other cell lines, but its $t \mathrm{BHQ}$-stimulated apoptosis was significantly decreased after intervention of $\mathrm{Nrfl}^{-/ \alpha^{-/}}$cells by this chemical for $4 \mathrm{~h}$ to $16 \mathrm{~h}$ (Fig. 6, A \& B), to a similar level to that of $t$ BHQ-treated $W T$ cells. This phenomenon appeared to be almost consistent with the results of changing ROS levels as detected above (Fig. 5, A to C). These indicate that $t \mathrm{BHQ}$ induces antioxidant cytoprotective response against endogenous oxidative stress arising from $\mathrm{Nrf}_{1} \alpha^{-/-}$cells, in which putative hyper-expressed Nrf2 may be allowed for a certain extent to ameliorate potential oxidative damage and apoptosis caused by loss of Nrf1 $\alpha$, but could not fully compensate the constitutive loss of Nrf1's function. Contrarily, no significant differences in basal apoptosis of $N r f 2^{-1-\Delta T A}$ or caNrf2 $2^{\Delta N}$ cell lines were observed when compared with that of WT cells (Fig. 6, A \& B), but both mutants led to a modest or less increase in $t$ BHQ-triggered apoptosis after intervention of $\mathrm{Nrf2}^{-/-\Delta T A}$ or $c a N r f 2^{\Delta N}$ cells, respectively. This indicates that the sensitivity to $t \mathrm{BHQ}$ cytotoxicity may be weakened by Nrf2 deficiency, allowing for the resistance of these two cell lines to a considerable extent of pro-oxidative stress. Overall, it could be concluded that both Nrf1 and Nrf2 play distinctive roles in mediating differential antioxidant cytoprotective responses against oxidative stress-induced apoptosis.

This concluding notion is supported by further luciferase reporter assays (Fig. 6C), in which the reporter gene was driven by two different ARE-battery sequences existing in the promoter region of human MT1E (i.e., MT1E-ARE1 and MT1E-ARE2). The results revealed that the transactivation activity of MT1E-2xARE1-luc was mediated by Nrf1 rather than Nrf2, but no changes in transcriptional expression of MT1E-2xARE2-luc were examined (Fig. 6D). However, a significant amplified activity of MT1E-6XARE2-luc was mediated by Nrf2 rather than Nrf1, even though the MT1E-6xARE1-luc was still modestly induced by Nrf1, but not Nrf2 (Fig. 6E). This difference in the activity of between MT1E-2XARE2-luc and MT1E-6xARE2-luc may be relevant to their contexts in the reporter gene constructs, but the detailed mechanism remains elusive.

\section{Discussion}

Since oxidative stress was initially formulated by Helmut Sies in 1985 and later redefined by Dean P. Jones in 2006 [46-48], an overwhelming number of publications by this conceptual term had been collected within at least 331,795 entries of the PubMed (https://pubmed.ncbi.nlm.nih.gov) until the middle of 2021. Such a continuously-heating topic on oxidative stress (and redox signaling) is open to arouse great concerns from researchers in distinct fields, but also is one of the most persistently-existing intractable problems to be addressed for health and disease, particularly in changing environmental conditions. Among its merits elicited by evoking biological stress responses, a steady-state redox balance is maintained within certain threshold ranges by cell respiration, aerobic metabolism and redox switches governing oxidative stress responses [46, 47]. However, the pitfalls of oxidative stress can also lead to indiscriminate use of this term as a global concept, but without a clear relation to redox chemistry, in each particular case. For the underlying molecular details, the major role in antioxidant defense is fulfilled by antioxidant enzymes, but not by small-molecule antioxidant compounds (e.g., $t \mathrm{BHQ})$, in the cellular biochemical processes. 
In all life forms, distinct cells can constantly generate a certain amount of ROS (and free radicals) during aerobic metabolism, so that its hormetic effects could be triggered in order to establish normal physiological cytoprotective mechanisms against oxidative damages. Of note, oxidative stress occurs in cells when ROS production overwhelms the natural antioxidant defenses and/or redox controls are disrupted [46, 47]. If oxidative stress is over-stimulated for a long term, the resulting damages lead to pathophysiological deterioration of many human chronic diseases, including cancer, diabetes, atherosclerosis, and neurodegenerative diseases [28, 49]. Thereby, to combat excessive production of ROS, all the cells have been evolutionarily armed with a series of innate powerful antioxidant defense systems. Among them is a set of essential antioxidant, detoxification and cytoprotective mechanisms governed by the CNC-bZIP family of transcription factors $[12,50,51]$. In mammalian cells, Nrf1 and Nrf2 are two principal CNC-bZIP factors to regulate target genes by specific ARE-binding sequences in their promoter regions. A large number of studies on Nrf2 revealed it functions as a master regulator of antioxidant response and relevant redox signaling [25]. Such versatile Nrf2 acts de facto as a promiscuous, but not essential player for ARE-binding to most of target genes [52], supporting the notion that Nrf2 is dispensable for normal growth and development [26]. As a matter of fact, Nrf1, rather than Nrf2, is a living fossil with its ancestral properties, because it shares a highly evolutionary conservativity with SKN-1, Cnc and Nach factors [51]. Like these ancient homologues [53, 54], Nrf1 is topologically dislocated across ER membranes and then processed to give rise to an N-terminally-truncated active factor, similar to Nrf2, before transactivating its target genes [55-57]. Hence, a unique conserved, indispensable role is fulfilled by Nrf1, but not by Nrf2, in maintaining the steady-state threshold of robust redox homeostasis.

The evidence has been provided in the present study, unraveling differential yet integral contributions of $\mathrm{Nrf1}$ and Nrf2 to synergistic regulation of antioxidant cytoprotective genes at basal and $t \mathrm{BHQ}$-inducible expression levels in wild-type (WT) cells. Specific knockout of $\mathrm{Nrf1}^{-/-}$leads to severe endogenous oxidative stress as elicited by increased basal ROS levels; this is accompanied by increased ratios of GSSG to GSH and apoptosis. In $\mathrm{Nrfl}^{-/-}$cells, Nrf2 was highly accumulated, but also cannot fully compensate loss of Nrf1 $\alpha^{\prime}$ s function in its basal cytoprotective response against endogenous oxidative stress, even though it had exerted partially inducible antioxidant response as the hormetic effect of $t \mathrm{BHQ}$ against apoptotic damages. By contrast, $\mathrm{Nrf2}{ }^{-/-\Delta T A}$ cells were manifested by obvious oxidative stress, partially resulting from a substantial reduction of $\mathrm{Nrf1}$ in basal and tBHQ-stimulated expression levels. However, the inactive $\mathrm{Nrf2} 2^{-/-\Delta T A}$ cells can be still triggered to mediate a potent antioxidant response to $t \mathrm{BHQ}$, as deciphered by a significantly decreased ration of GSSG to GSH. Conversely, a remarkable increase of the Nrf1 expression was obtained from the constitutive active caNrf $2^{\Delta N}$ cells, in which neither oxidative stress nor apoptotic damages had occurred, no matter if it was intervened with $t \mathrm{BHQ}$. Thereby, distinct yet joint functions of Nrf1 and Nrf2 may be executed through their inter-regulatory effects on cognate genes against oxidative stress (Fig. 6F).

Differences in ROS-scavenging activities of SOD and CAT were determined in distinct genotypic cell lines. Basal activities of SOD and CAT were significantly increased by $\mathrm{Nrf1} \alpha^{-/-}, \mathrm{Nrf2} 2^{-1-\Delta T A}$ or caNrf2 ${ }^{\triangle N}$, when compared to

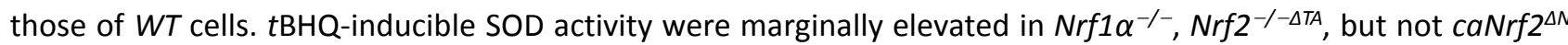
or $W T$, cell lines, as accompanied by an exceptional decrease of its activity by $16 \mathrm{~h}$ of this stimulation. The modest changes suggest that SOD activity may be monitored by other factors beyond Nrf1 and Nrf2. This notion is also supported by the previous RT-qPCR data [45]. Further evidence also revealed that $t$ BHQ-stimulated CAT activity was markedly augmented in $\mathrm{Nrf1}^{-/-}$cells (with hyper-active Nrf2 accumulation), and thereby completely

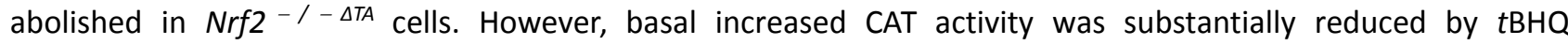
intervention of caNrf2 $2^{\Delta N}$ cells (with the enhanced expression of Nrf1), although this constitutive activator per se was unaffected by this chemical. These imply that CAT activity is regulated positively by Nrf2, and also monitored negatively by Nrf1, in particularly $t \mathrm{BHQ}$-stimulated conditions. As such, it cannot also be ruled out that loss of the $\mathrm{N}$-terminal Keap1-binding Neh2 domain from Nrf2 to yield caNrf2 ${ }^{\Delta N}$ causes a negative effect on the $t$ BHQ-stimulated CAT activity. 
As a widely used Nrf2-activator, $t \mathrm{BHQ}$ can also trigger a certain activating effect on the expression of Nrf1 in $W T$ cells, as well in caNrf2 $2^{\Delta N}$ cells, but this effect is almost abolished by $N r f 1 \alpha^{-/-}$or $N r f 2^{-/-\Delta T A}$. Conversely, induction of $\mathrm{Nrf2}$ expression by $t \mathrm{BHQ}$ occurred only in $\mathrm{WT}$ cells, but not in $\mathrm{Nrf1} \alpha^{-/-}, \mathrm{Nrf2} 2^{-/-\Delta T A}$ or caNrf2 ${ }^{\triangle N}$ cell lines, although its basal expression levels are significantly augmented in $N r f 1 \alpha^{-/-}$or caNrf2 ${ }^{\Delta N}$ cell lines. Together, with our previous data $[31,40]$, these indicate that Nrf1 has an ability to confine Nrf2 within a certain extent, albeit its transcriptional expression is positively regulated by Nrf2, which functions as a limited chameleon activator. Such inter-regulatory effects of Nrf1 and Nrf2 on antioxidant, detoxification and cytoprotective genes, such as HO-1, NQO1, GCLC, GCLM, GSR, GPX1, TALDO, MT1E and MT2, were further determined in distinct genotypic cell lines. As anticipated, the comprehensive experimental evidence has been provided herein, unraveling that HO-1, NQO1, GCLC, GCLM, GSR and TALDO were induced by $t B H Q$ stimulation of WT cells, but GPX1, MT1E and MT2 were not stimulated or even slightly suppressed by this chemical. By contrast, Nrf1 $\alpha^{-/-}$ cells were still allowed for $t \mathrm{BHQ}$-increased expression of HO-1, NQO1, GCLM and TALDO, but with an exceptional decrease of GPX1, even though hyper-expressed Nrf2 was unaffected by $t B H Q$. This implies an additional involvement of other transcriptional factors beyond $\mathrm{Nrf1}$ and $\mathrm{Nrf2}$ in these gene response to $t \mathrm{BHQ}$ as a pro-oxidative stressor. Intriguingly, both basal and $t B H Q$-stimulated expression levels of MT1E were strikingly augmented in $N r f 2^{-/-\Delta T A}$, but not $N r f 1 \alpha^{-/-}$or caNrf2 $2^{\Delta N}$, cell lines, whereas $M T 2$ was marginally induced by $t B H Q$ in caNrf2 ${ }^{\Delta N}$ cells. This finding implies that MT1E, but not MT2, may serve as an Nrf1-specific target gene, as further evidenced by its relevant reporter assays. Moreover, these was accompanied by a modest inducible enhancement of GLCM and Nrf1, whereas all other examined genes were, to lesser or no extents, stimulated by $t \mathrm{BHQ}$ in either $N r f 2^{-/-\triangle T A}$ or caNrf2 $2^{\Delta N}$ cell lines. These indicated that most of all other examined genes except MT1E are regulated primarily by Nrf2, but induction of its transactivation activity by $t \mathrm{BHQ}$ is also limited by its constitutive loss of the Keap1-binding Neh2 domain in the mutant caNrf2 ${ }^{\Delta N}$ factor.

\section{Concluding remarks}

Dramatic research advances of the past 25 years, since a fascinating discovery by Itoh, et al [23], have witnessed an overwhelmingly preferential option for Nrf2 lonely focused in all relevant fields, whereas Nrf1 was almost totally ignored by all others except for a very few of groups. Such disproportionately-biased consequence has resulted in a general misunderstanding of Nrf2 as an only master hub of predominantly regulating antioxidant, detoxification and cytoprotective genes, regardless of the exiting facts that Nrf1, rather than Nrf2, is highly conserved with those more ancient SKN-1, Cnc and Nach factors [51], and can fulfill unique indispensable roles for cell homeostasis and organ integrity during life process. In the present study, together with our previous publications [31, 40], Nrf1 and Nrf2 are experimentally evidenced to elicit differential yet integral roles in mediating antioxidant cytoprotective responsive genes against pro-oxidative stress induced by $t \mathrm{BHQ}$. The inter-regulatory effects of Nrf1 and Nrf2 on differential expression levels of antioxidant cytoprotective genes, e.g., HO-1, NQO1, GCLC, GCLM, GSR, GPX1, TALDO, MT1E and MT2, as well on the ROS-scavenging activities of SOD and CAT, were determined in depth. The collective results demonstrate that both Nrf1 and Nrf2 can make distinctive yet cooperative contributions to finely tuning basal and $t \mathrm{BHQ}$-stimulated expression of target genes within the inter-regulatory networks. Overall, Nrf1 is allowed to act as a brake control for confining Nrf2's functionality within a certain extent, albeit its transcriptional expression is positively regulated by Nrf2.

Author contributions: Both Z.W. and Z. F. performed the experiments with help of K.L., collected all the relevant data, made draft of this manuscript with most figures and supplemental information. Y.Z. designed and supervised this study, analyzed all the data, helped to prepare all figures with cartoons, wrote and revised the paper.

Acknowledgments: We are greatly thankful to Drs. Lu Qiu (Zhengzhou University, Henan, China) and Yonggang Ren (North Sichuan Medical College, Sichuan, China) for their involvement in establishing the indicated cell lines used in this study. We also thank to all other present and past members of Prof. Zhang's laboratory (at Chongqing University, China) for giving critical discussion and invaluable help with this work. Notably, this study was funded by the National Natural Science 
Foundation of China (NSFC, with a key program 91429305 and other two projects 81872336 and 82073079 ) awarded to Prof. Yiguo Zhang.

\section{References}

1. Shahidi, F. (2000) Antioxidants in food and food antioxidants, Nahrung. 44, 158-63.

2. Oswell, N. J., Thippareddi, H. \& Pegg, R. B. (2018) Practical use of natural antioxidants in meat products in the U.S.: A review, Meat science. 145, 469-479.

3. Conning, D. M. \& Phillips, J. C. (1986) Comparative metabolism of BHA, BHT and other phenolic antioxidants and its toxicological relevance, Food Chem Toxicol. 24, 1145-8.

4. van Esch, G. J. (1986) Toxicology of tert-butylhydroquinone (TBHQ), Food Chem Toxicol. 24, 1063-5.

5. Nakamura, Y., Kumagai, T., Yoshida, C., Naito, Y., Miyamoto, M., Ohigashi, H., Osawa, T. \& Uchida, K. (2003) Pivotal role of electrophilicity in glutathione S-transferase induction by tert-butylhydroquinone, Biochemistry. 42, 4300-9.

6. Gharavi, N., Haggarty, S. \& El-Kadi, A. O. (2007) Chemoprotective and carcinogenic effects of tert-butylhydroquinone and its metabolites, Curr Drug Metab. 8, 1-7.

7. Gharavi, N. \& El-Kadi, A. O. (2005) tert-Butylhydroquinone is a novel aryl hydrocarbon receptor ligand, Drug Metab Dispos. 33, 365-72.

8. Xiao, H., Lu, F., Stewart, D. \& Zhang, Y. (2013) Mechanisms underlying chemopreventive effects of flavonoids via multiple signaling nodes within Nrf2-ARE and AhR-XRE gene regulatory networks., Curr Chem Biol. 7, 151-176.

9. Xu, C., Li, C. Y. \& Kong, A. N. (2005) Induction of phase I, II and III drug metabolism/transport by xenobiotics, Arch Pharm Res. 28, 249-68.

10. Deneke, S. M. (2000) Thiol-based antioxidants, Curr Top Cell Regul. 36, 151-80.

11. Yamamoto, M., Kensler, T. W. \& Motohashi, H. (2018) The KEAP1-NRF2 System: a Thiol-Based Sensor-Effector Apparatus for Maintaining Redox Homeostasis, Physiol Rev. 98, 1169-1203.

12. Zhang, Y. \& Xiang, Y. (2016) Molecular and cellular basis for the unique functioning of Nrf1, an indispensable transcription factor for maintaining cell homoeostasis and organ integrity, The Biochemical journal. 473, $961-1000$.

13. Sant, K. E., Hansen, J. M., Williams, L. M., Tran, N. L., Goldstone, J. V., Stegeman, J. J., Hahn, M. E. \& Timme-Laragy, A. (2017) The role of Nrf1 and Nrf2 in the regulation of glutathione and redox dynamics in the developing zebrafish embryo, Redox Biol. 13, 207-218.

14. Leung, L., Kwong, M., Hou, S., Lee, C. \& Chan, J. Y. (2003) Deficiency of the Nrf1 and Nrf2 transcription factors results in early embryonic lethality and severe oxidative stress, J Biol Chem. 278, 48021-9.

15. Yang, H., Magilnick, N., Lee, C., Kalmaz, D., Ou, X., Chan, J. Y. \& Lu, S. C. (2005) Nrf1 and Nrf2 Regulate Rat Glutamate-Cysteine Ligase Catalytic Subunit Transcription Indirectly via NF-\{kappa\}B and AP-1, Mol Cell Biol. 25 , 5933-46.

16. Ohtsuji, M., Katsuoka, F., Kobayashi, A., Aburatani, H., Hayes, J. D. \& Yamamoto, M. (2008) Nrf1 and Nrf2 play distinct roles in activation of antioxidant response element-dependent genes, J Biol Chem. 283, 33554-62.

17. Nouhi, F., Tusi, S. K., Abdi, A. \& Khodagholi, F. (2011) Dietary supplementation with tBHQ, an Nrf2 stabilizer molecule, confers neuroprotection against apoptosis in amyloid beta-injected rat, Neurochem Res. 36, 870-8.

18. Lau, A., Whitman, S. A., Jaramillo, M. C. \& Zhang, D. D. (2012) Arsenic-mediated activation of the Nrf2-Keap1 antioxidant pathway, J Biochem Mol Toxicol. 27, 99-105.

19. Parastan, R. H., Christopher, M., Torrys, Y. S. \& Mahadewa, T. G. B. (2020) Combined Therapy Potential of Apocynin and Tert-butylhydroquinone as a Therapeutic Agent to Prevent Secondary Progression to Traumatic Brain Injury, Asian journal of neurosurgery. 15, 10-15.

20. Kwong, M., Kan, Y. W. \& Chan, J. Y. (1999) The CNC basic leucine zipper factor, Nrf1, is essential for cell survival in response to oxidative stress-inducing agents. Role for Nrf1 in gamma-gcs(I) and gss expression in mouse fibroblasts, $J$ Biol Chem. 274, 37491-8.

21. Chen, L., Kwong, M., Lu, R., Ginzinger, D., Lee, C., Leung, L. \& Chan, J. Y. (2003) Nrf1 is critical for redox balance and survival of liver cells during development, Mol Cell Biol. 23, 4673-86. 
22. Xu, Z., Chen, L., Leung, L., Yen, T. S., Lee, C. \& Chan, J. Y. (2005) Liver-specific inactivation of the Nrf1 gene in adult mouse leads to nonalcoholic steatohepatitis and hepatic neoplasia, Proc Natl Acad Sci U S A. 102, 4120-5.

23. Itoh, K., Chiba, T., Takahashi, S., Ishii, T., Igarashi, K., Katoh, Y., Oyake, T., Hayashi, N., Satoh, K., Hatayama, I., Yamamoto, M. \& Nabeshima, Y. (1997) An Nrf2/small Maf heterodimer mediates the induction of phase II detoxifying enzyme genes through antioxidant response elements, Biochem Biophys Res Commun. 236, 313-22.

24. Baird, L. \& Yamamoto, M. (2020) The Molecular Mechanisms Regulating the KEAP1-NRF2 Pathway, Mol Cell Biol. 40.

25. Cuadrado, A., Rojo, A. I., Wells, G., Hayes, J. D., Cousin, S. P., Rumsey, W. L., Attucks, O. C., Franklin, S., Levonen, A. L., Kensler, T. W. \& Dinkova-Kostova, A. T. (2019) Therapeutic targeting of the NRF2 and KEAP1 partnership in chronic diseases, Nat Rev Drug Discov. 18, 295-317.

26. Chan, K., Lu, R., Chang, J. C. \& Kan, Y. W. (1996) NRF2, a member of the NFE2 family of transcription factors, is not essential for murine erythropoiesis, growth, and development, Proc Natl Acad Sci U S A. 93, 13943-8.

27. Xu, C., Huang, M. T., Shen, G., Yuan, X., Lin, W., Khor, T. O., Conney, A. H. \& Kong, A. N. (2006) Inhibition of 7,12-dimethylbenz(a)anthracene-induced skin tumorigenesis in C57BL/6 mice by sulforaphane is mediated by nuclear factor E2-related factor 2, Cancer Res. 66, 8293-6.

28. Hayes, J. D., Dinkova-Kostova, A. T. \& Tew, K. D. (2020) Oxidative Stress in Cancer, Cancer Cell. 38, 167-197.

29. Hayes, J. D., McMahon, M., Chowdhry, S. \& Dinkova-Kostova, A. T. (2010) Cancer chemoprevention mechanisms mediated through the Keap1-Nrf2 pathway, Antioxid Redox Signal. 13, 1713-48.

30. Rojo de la Vega, M., Chapman, E. \& Zhang, D. D. (2018) NRF2 and the Hallmarks of Cancer, Cancer Cell.

31. Qiu, L., Wang, M., Hu, S., Ru, X., Ren, Y., Zhang, Z., Yu, S. \& Zhang, Y. (2018) Oncogenic Activation of Nrf2, Though as a Master Antioxidant Transcription Factor, Liberated by Specific Knockout of the Full-Length Nrf1alpha that Acts as a Dominant Tumor Repressor, Cancers. 10.

32. Yuan, J., Zhang, S. \& Zhang, Y. (2018) Nrf1 is paved as a new strategic avenue to prevent and treat cancer, neurodegenerative and other diseases, Toxicol Appl Pharmacol. 360, 273-283.

33. Chen, J., Wang, M., Xiang, Y., Ru, X., Ren, Y., Liu, X., Qiu, L. \& Zhang, Y. (2020) Nrf1 Is Endowed with a Dominant Tumor-Repressing Effect onto the Wnt/beta-Catenin-Dependent and Wnt/beta-Catenin-Independent Signaling Networks in the Human Liver Cancer, Oxid Med Cell Longev. 2020, 5138539.

34. Farmer, S. C., Sun, C. W., Winnier, G. E., Hogan, B. L. \& Townes, T. M. (1997) The bZIP transcription factor LCR-F1 is essential for mesoderm formation in mouse development, Genes Dev. 11, 786-98.

35. Chan, J. Y., Kwong, M., Lu, R., Chang, J., Wang, B., Yen, T. S. \& Kan, Y. W. (1998) Targeted disruption of the ubiquitous CNC-bZIP transcription factor, Nrf-1, results in anemia and embryonic lethality in mice, Embo J. 17, $1779-87$.

36. Tsujita, T., Peirce, V., Baird, L., Matsuyama, Y., Takaku, M., Walsh, S. V., Griffin, J. L., Uruno, A., Yamamoto, M. \& Hayes, J. D. (2014) Transcription factor Nrf1 negatively regulates the cystine/glutamate transporter and lipid-metabolizing enzymes, Mol Cell Biol. 34, 3800-16.

37. Zheng, H., Fu, J., Xue, P., Zhao, R., Dong, J., Liu, D., Yamamoto, M., Tong, Q., Teng, W., Qu, W., Zhang, Q., Andersen, M. E. \& Pi, J. (2015) CNC-bZIP protein Nrf1-dependent regulation of glucose-stimulated insulin secretion, Antioxid Redox Signal. 22, 819-31.

38. Kobayashi, A., Tsukide, T., Miyasaka, T., Morita, T., Mizoroki, T., Saito, Y., Ihara, Y., Takashima, A., Noguchi, N., Fukamizu, A., Hirotsu, Y., Ohtsuji, M., Katsuoka, F. \& Yamamoto, M. (2011) Central nervous system-specific deletion of transcription factor Nrf1 causes progressive motor neuronal dysfunction, Genes Cells. 16, 692-703.

39. Lee, C. S., Lee, C., Hu, T., Nguyen, J. M., Zhang, J., Martin, M. V., Vawter, M. P., Huang, E. J. \& Chan, J. Y. (2011) Loss of nuclear factor E2-related factor 1 in the brain leads to dysregulation of proteasome gene expression and neurodegeneration, Proc Natl Acad Sci U S A. 108, 8408-13.

40. Zhu, Y. P., Zheng, Z., Hu, S., Ru, X., Fan, Z., Qiu, L. \& Zhang, Y. (2019) Unification of Opposites between Two Antioxidant Transcription Factors Nrf1 and Nrf2 in Mediating Distinct Cellular Responses to the Endoplasmic Reticulum Stressor Tunicamycin, Antioxidants. 9. 
41. Xiang, Y., Wang, M., Hu, S., Qiu, L., Yang, F., Zhang, Z., Yu, S., Pi, J. \& Zhang, Y. (2018) Mechanisms controlling the multistage post-translational processing of endogenous Nrf1alpha/TCF11 proteins to yield distinct isoforms within the coupled positive and negative feedback circuits, Toxicol Appl Pharmacol. 360, 212-235.

42. Hanczko, R., Fernandez, D. R., Doherty, E., Qian, Y., Vas, G., Niland, B., Telarico, T., Garba, A., Banerjee, S., Middleton, F. A., Barrett, D., Barcza, M., Banki, K., Landas, S. K. \& Perl, A. (2009) Prevention of hepatocarcinogenesis and increased susceptibility to acetaminophen-induced liver failure in transaldolase-deficient mice by $\mathrm{N}$-acetylcysteine, The Journal of clinical investigation. 119, 1546-57.

43. Pavic, M., Turcic, P. \& Ljubojevic, M. (2019) Forgotten partners and function regulators of inducible metallothioneins, Arhiv za higijenu rada i toksikologiju. 70, 256-264.

44. Eruslanov, E. \& Kusmartsev, S. (2010) Identification of ROS using oxidized DCFDA and flow-cytometry, Methods Mol Biol. 594, 57-72.

45. Zhu, Y. P., Zheng, Z., Xiang, Y. \& Zhang, Y. (2020) Glucose Starvation-Induced Rapid Death of Nrf1alpha-Deficient, but Not Nrf2-Deficient, Hepatoma Cells Results from Its Fatal Defects in the Redox Metabolism Reprogramming, Oxid Med Cell Longev. 2020, 4959821.

46. Sies, H. (2015) Oxidative stress: a concept in redox biology and medicine, Redox Biol. 4, 180-3.

47. Sies, H., Berndt, C. \& Jones, D. P. (2017) Oxidative Stress, Annu Rev Biochem. 86, 715-748.

48. Jones, D. P. (2006) Redefining oxidative stress, Antioxid Redox Signal. 8, 1865-79.

49. Zhang, Y. (2009) Molecular and cellular control of the Nrf1 transcription factor: An integral membrane glycoprotein, Vdm Verlag Dr Müller Publishing House. Germany (May 2009). The first edition pp1-264.

50. Sykiotis, G. P. \& Bohmann, D. (2010) Stress-activated cap'n'collar transcription factors in aging and human disease, Sci Signal. 3, re3.

51. Zhu, Y. P., Wang, M., Xiang, Y., Qiu, L., Hu, S., Zhang, Z., Mattjus, P., Zhu, X. \& Zhang, Y. (2018) Nach Is a Novel Subgroup at an Early Evolutionary Stage of the CNC-bZIP Subfamily Transcription Factors from the Marine Bacteria to Humans, Int J Mol Sci. 19, 1-26.

52. Liu, P., Kerins, M. J., Tian, W., Neupane, D., Zhang, D. D. \& Ooi, A. (2019) Differential and overlapping targets of the transcriptional regulators NRF1, NRF2, and NRF3 in human cells, J Biol Chem. 294, 18131-18149.

53. Lehrbach, N. J. \& Ruvkun, G. (2019) Endoplasmic reticulum-associated SKN-1A/Nrf1 mediates a cytoplasmic unfolded protein response and promotes longevity, Elife. $\mathbf{8}$.

54. Karim, M. R., Taniguchi, H. \& Kobayashi, A. (2015) Constitutive activation of Drosophila CncC transcription factor reduces lipid formation in the fat body, Biochem Biophys Res Commun. 463, 693-8.

55. Zhang, Y., Ren, Y., Li, S. \& Hayes, J. D. (2014) Transcription factor Nrf1 is topologically repartitioned across membranes to enable target gene transactivation through its acidic glucose-responsive domains, PLoS One. 9, e93458.

56. Xiang, Y., Halin, J., Fan, Z., Hu, S., Wang, M., Qiu, L., Zhang, Z., Mattjus, P. \& Zhang, Y. (2018) Topovectorial mechanisms control the juxtamembrane proteolytic processing of Nrf1 to remove its N-terminal polypeptides during maturation of the CNC-bZIP factor, Toxicol Appl Pharmacol. 360, 160-184.

57. Wang, M., Ren, Y., Hu, S., Liu, K., Qiu, L. \& Zhang, Y. (2021) TCF11 Has a Potent Tumor-Repressing Effect than Its Prototypic Nrf1 $\alpha$ by Definition of both Similar yet Different Regulatory Profiles, with a Striking Disparity from Nrf2, bioRxiv. doi: https://doi.org/10.1101/2021.01.12.426360. 
Figure legends

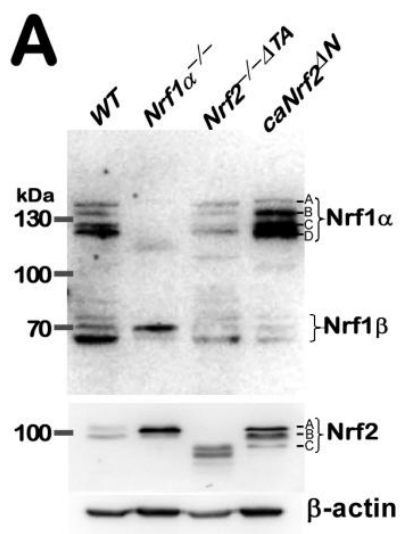

\section{B}

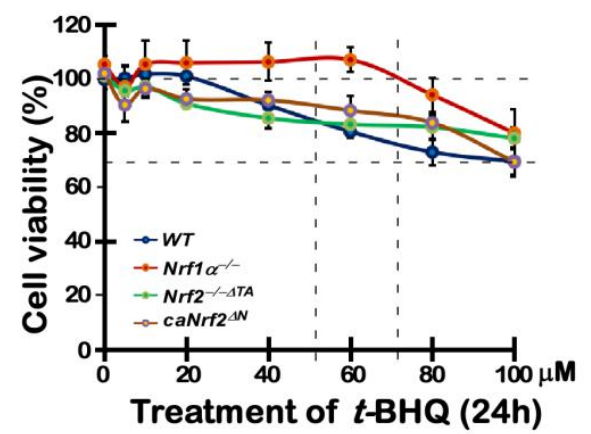

C

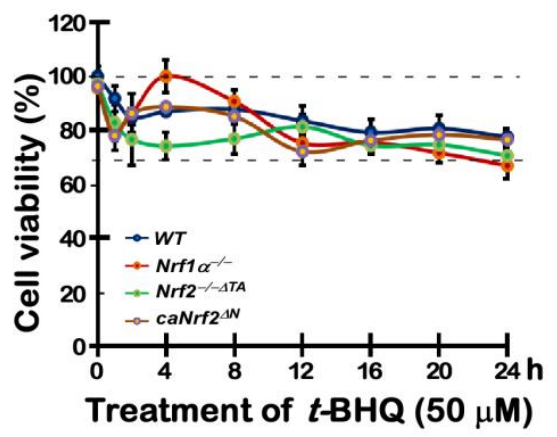

D

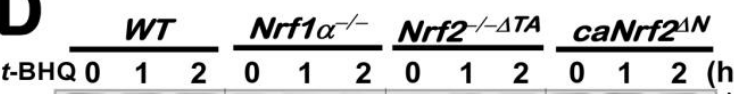

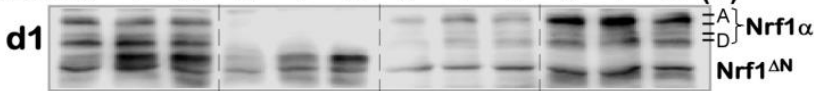

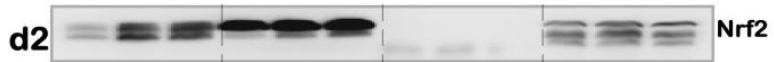

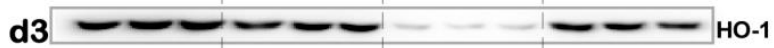

d4 - - - NQO1 d5 $\beta$-actin

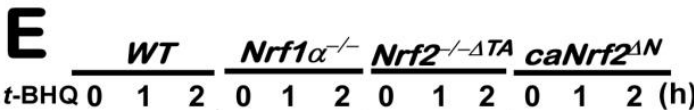

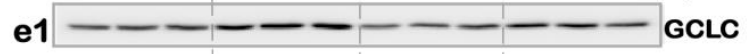

$\mathrm{e2}$

e3- - - - - - - -

e4 - -

e5 $\approx==\mathrm{E}=\mathrm{E}=\mathrm{TALDO1}$

e6 - $\beta$-actin

Figure 1. Distinct effects of $t \mathrm{BHQ}$ on different cell viability and antioxidant responsive genes.

(A) Distinct protein abundances of $\mathrm{Nrf1}$ and $\mathrm{Nrf2}$ in $\mathrm{WT}, \mathrm{Nrf1} \alpha^{-{ }^{--}}, \mathrm{Nrf2} 2^{-/-\Delta T A}$ or caNrf2 ${ }^{\Delta N}$ cell lines were determined by western blotting with their specific antibodies. (B \& C) Different cell viability was examined by the MTT- based assay, after the indicated cells had been treated with $t \mathrm{BHQ}:(B)$ at different doses (from 0 to $100 \mu \mathrm{M}$ ) for $24 \mathrm{~h} ;(C)$ at a single dose of 50 $\mu \mathrm{M}$ for different length of time. (D \& E) Four distinct cell lines were treated with $50 \mu \mathrm{M} t \mathrm{BHQ}$ or not for a short time (from 0 to $2 \mathrm{~h}$ ), followed by western blotting of Nrf1, Nrf2 and other ARE-driven target gene expression levels as indicated. 
$04812162024 \mathrm{~h}$ after $t$-BHQ treatment in four different cell lines
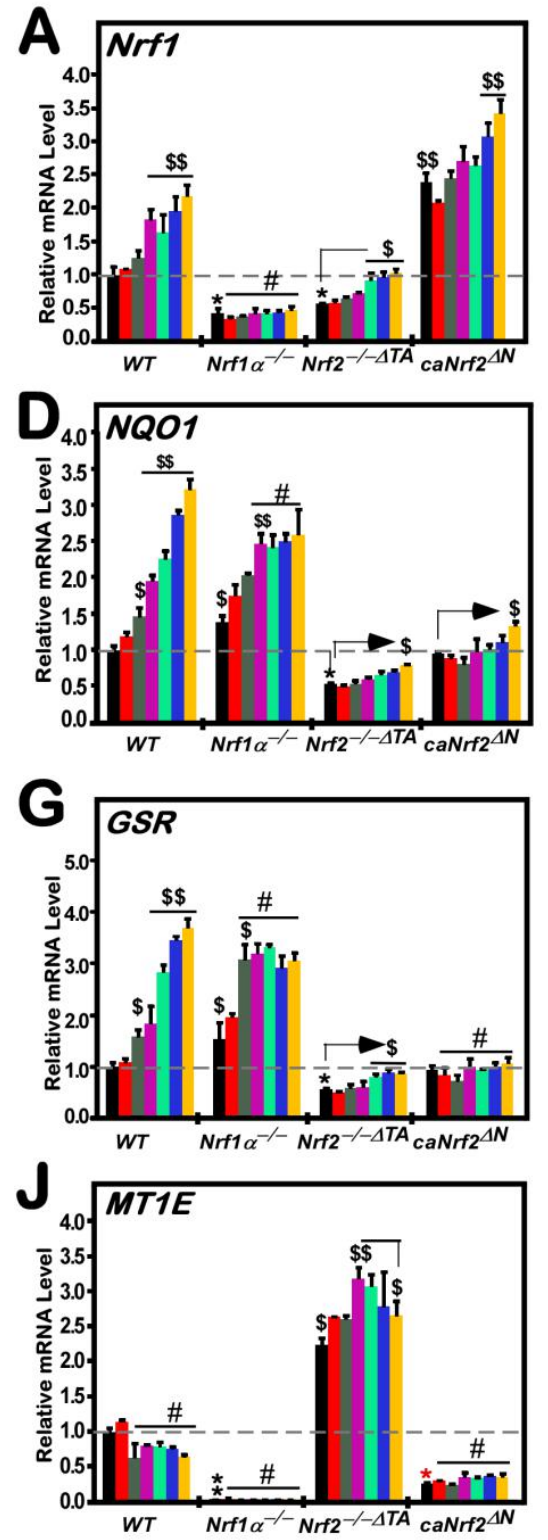
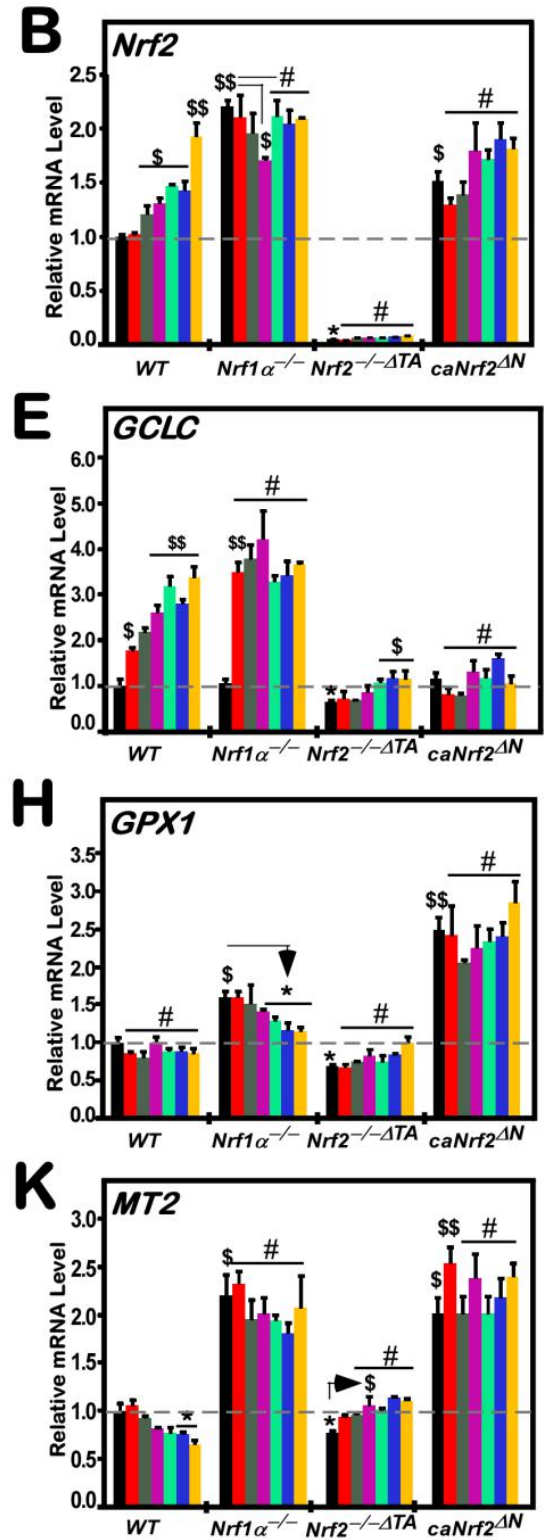
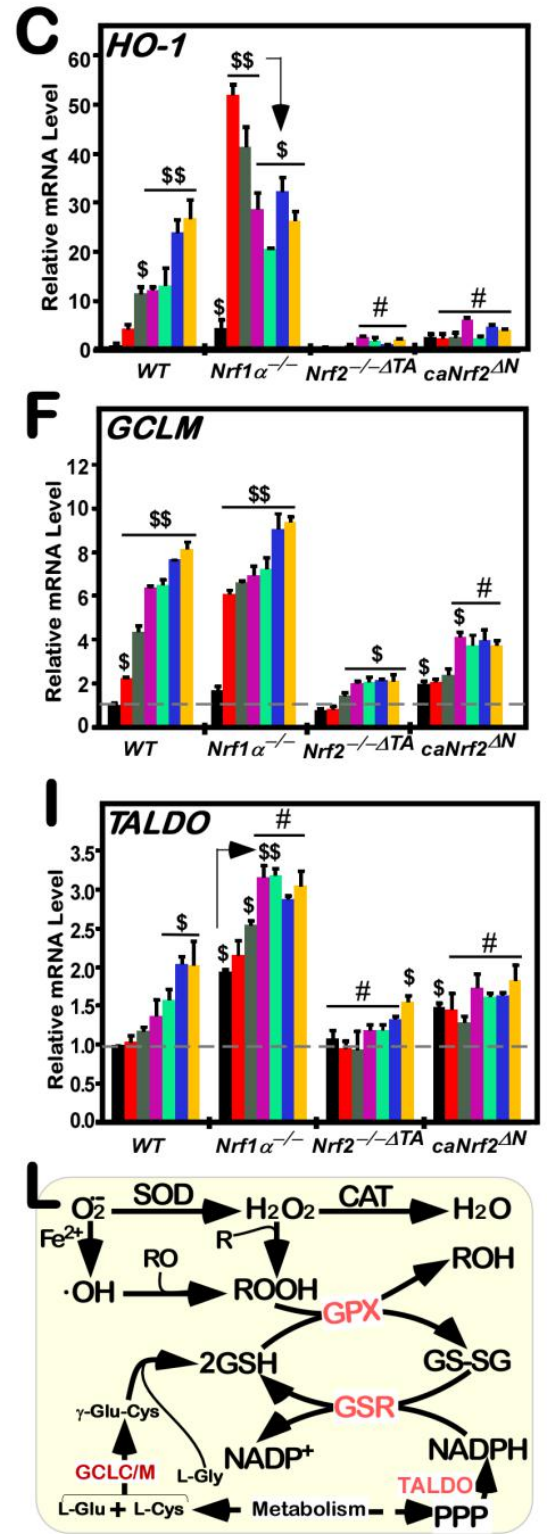

Figure 2. Time-dependent changes in the mRNA expression of distinctive responsive genes to tBHQ.

Distinct genotypic cell lines of $W T, N r f 1 \alpha^{-{ }^{-}}, N r f 2^{-1-\Delta T A}$ or caNrf2 $2^{\Delta N}$ or were not treated with $50 \mu \mathrm{M} t \mathrm{BHQ}$ for 0 to $24 \mathrm{~h}$, before both basal and $t B H Q$-inducible mRNA expression levels of all examined genes were determined by RT-qPCR. These genes included Nrf1 (A), Nrf2 (B), HO-1 (C), NQO1 (D), GCLC (E), GCLM (F), GSR (G), GPX1 (H), TALDO (I), MT1E (J) and MT2 (K). Then, statistic significances were calculated as described in "Materials and Methods". Significant increases $(\$, p<0.05 ;$ $\$ \$, p<0.01)$, and significant decreases $\left({ }^{*} P<0.05 ;{ }^{*} P<0.01\right)$, were indicated, respectively, in addition to the non-significance (\#). (L) A schematic representation of major ROS-scavenging enzymes and relevant redox signaling. 
A

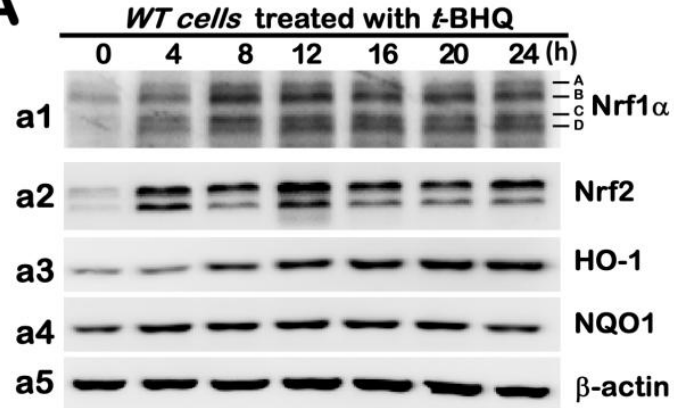

B

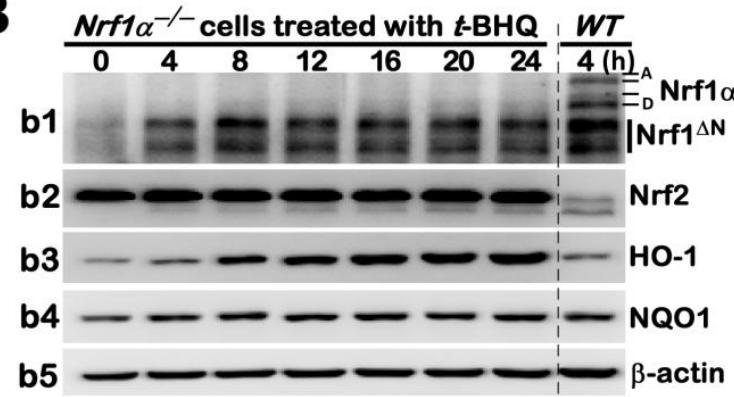

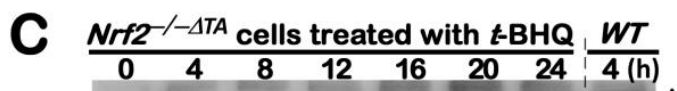
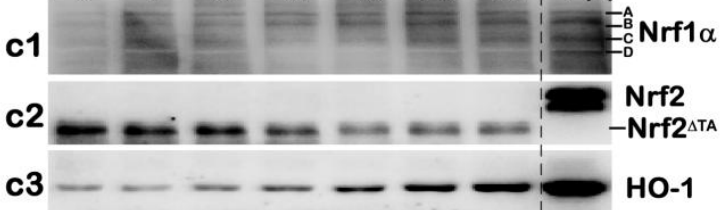

c4 - - - - NQ01

c5

$\beta$-actin

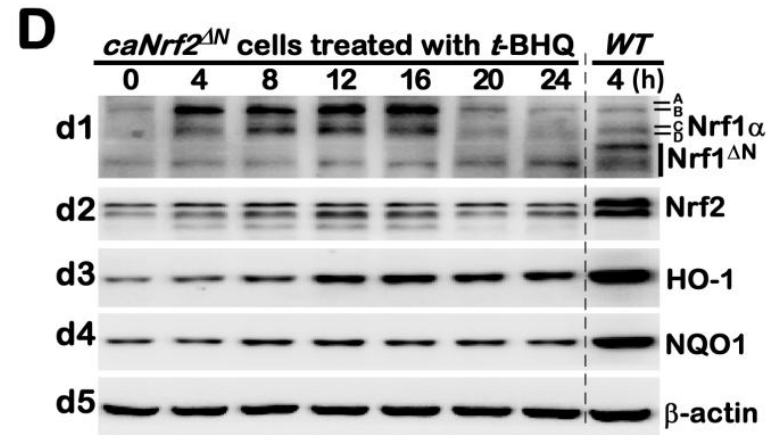

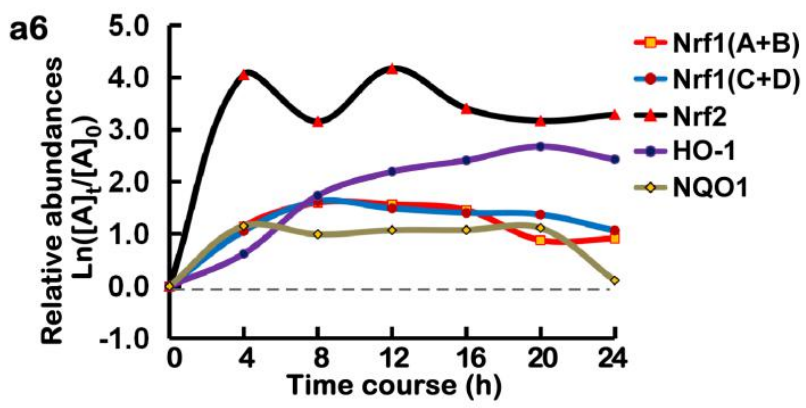
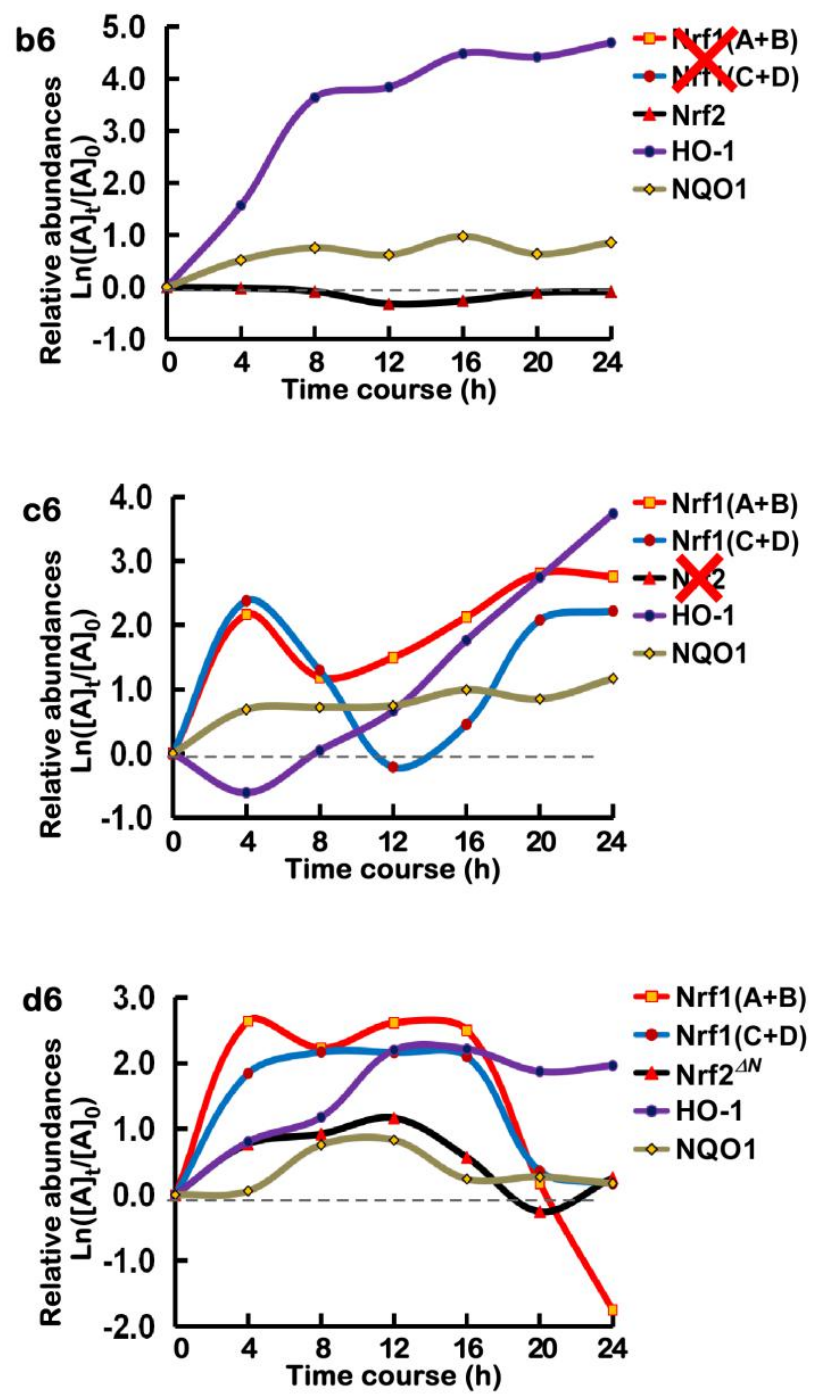

Figure 3. Long-term effects of $t \mathrm{BHQ}$ on protein abundances of Nrf1, Nrf2 and co-target genes.

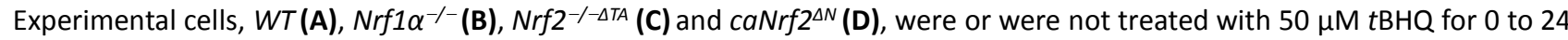
$\mathrm{h}$, before basal and $t \mathrm{BHQ}$-inducible protein changes of Nrf1, Nrf2, HO-1 and NQO1 were determined by western blotting with their indicated antibodies, whilst $\beta$-actin served as a loading control. The intensity of those immunoblots representing different protein expression levels was also quantified by the Quantity One 4.5.2 software (Bio-rad, Hercules, CA, USA), and then shown graphically (in right panels). In addition, it should be noted that two big red crosses represent the constitutive losses of Nrf1 or Nrf2 (b5 and c5), respectively. 
A

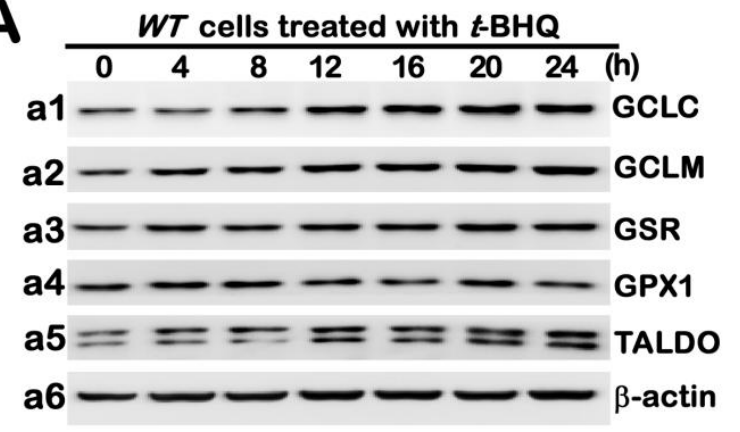

B \begin{tabular}{ccccccc:c} 
& $N r f 1 \alpha^{-/-}$cells treated with $t$-BHQ & WT \\
\hdashline 0 & 4 & 8 & 12 & 16 & 20 & 24 & $4(\mathrm{~h})$
\end{tabular} b1 - - - - - - GCLC b2 - - - - - GCLM b3 - - - - - - GSR b4 - - - - - GPX1 $\mathrm{b5}===-\overline{=}=\mathrm{TALDO}$ b6 - - - - - - $-1-$-actin

\section{C $\begin{array}{ccccccc:c}N_{r f 2^{-}}-\Delta T A & & & & \\ 0 & 4 & 8 & 12 & 16 & 20 & 24 & 4(\mathrm{~h})\end{array}$}

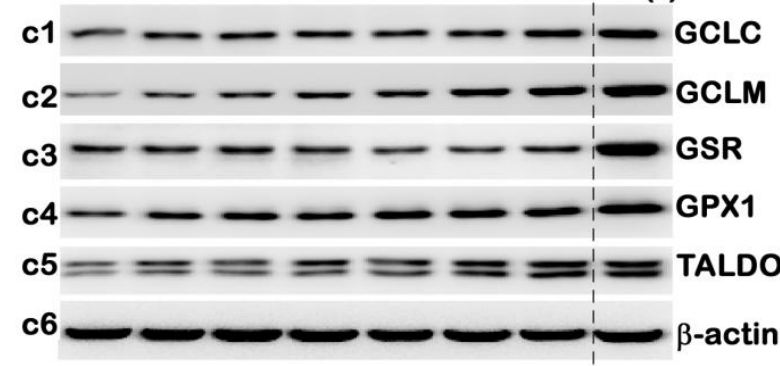

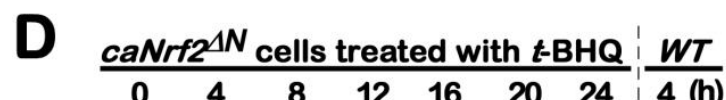

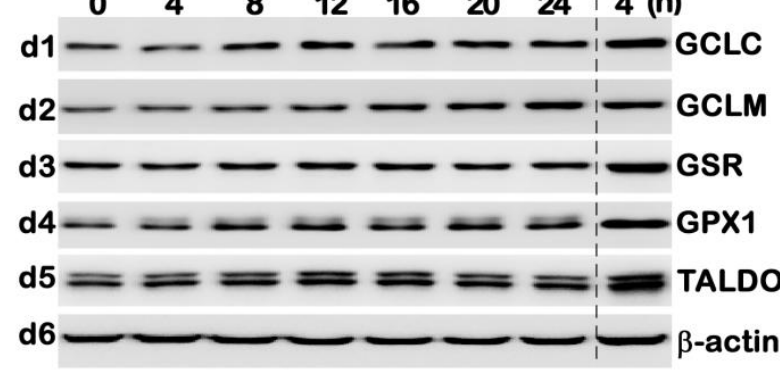

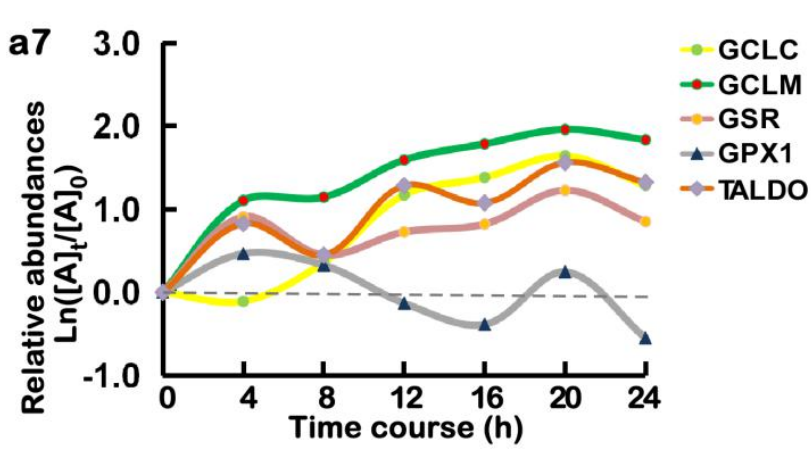
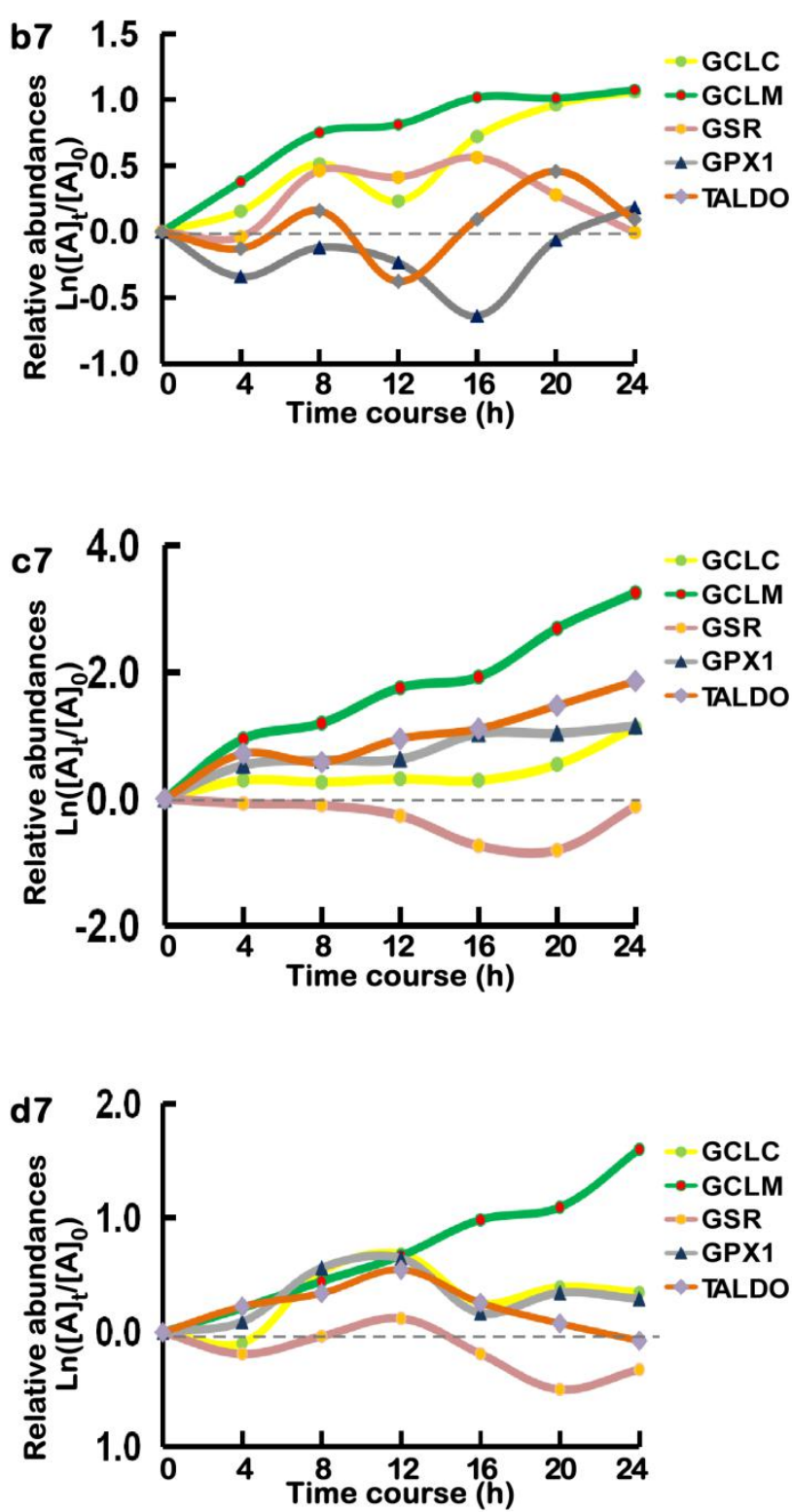

Figure 4. Long-term $t \mathrm{BHQ}$-stimulated changes in the protein expression of antioxidant responsive genes.

Different lines of $W T(A), N r f 1 \alpha^{-/-}$(B), Nrf2 $2^{-/-\Delta T A}$ (C) and caNrf2 ${ }^{\Delta N}$ (D) were treated with $50 \mu \mathrm{M} t \mathrm{BHQ}$ or not for 0 to $24 \mathrm{~h}$, before basal and stimulated abundances of those antioxidant cytoprotective proteins, e.g., GCLC, GCLM, GSR, GPX1 and TALDO, were determined by western blotting with the indicated antibodies. The intensity of relevant immunoblots representing different protein expression levels was quantified by the Quantity One 4.5 .2 software, and then shown graphically. 


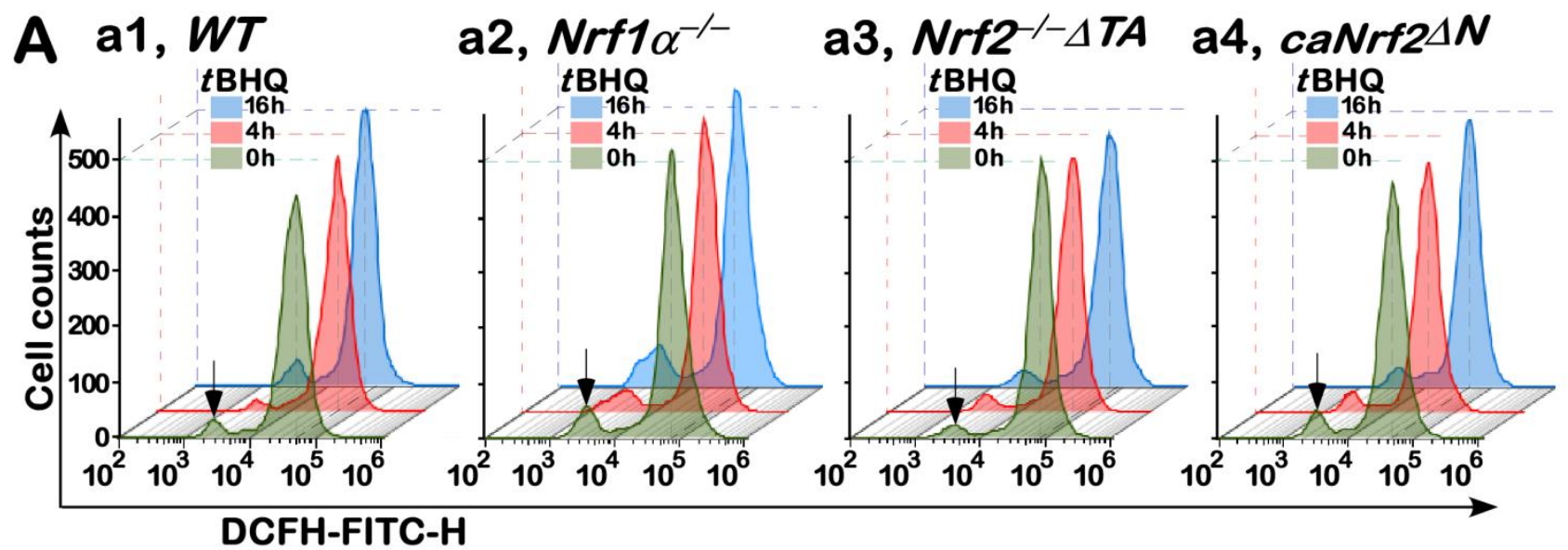

B

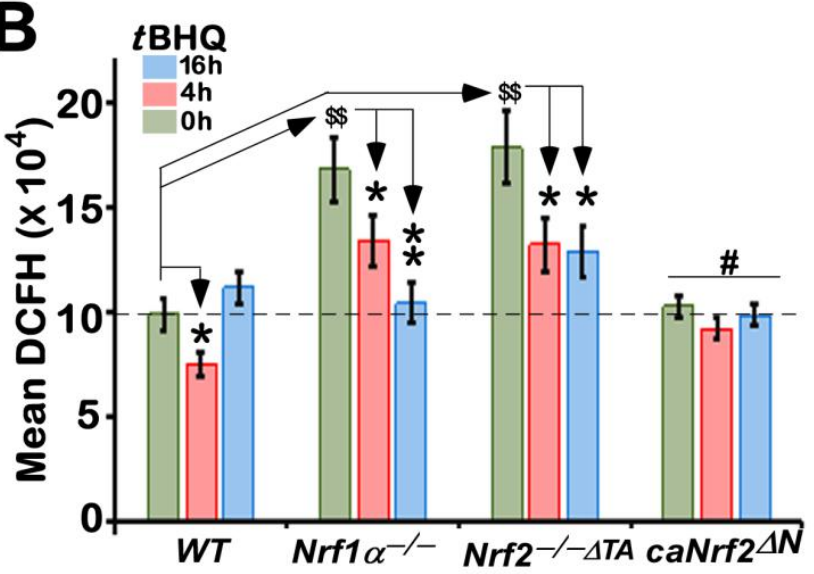

D

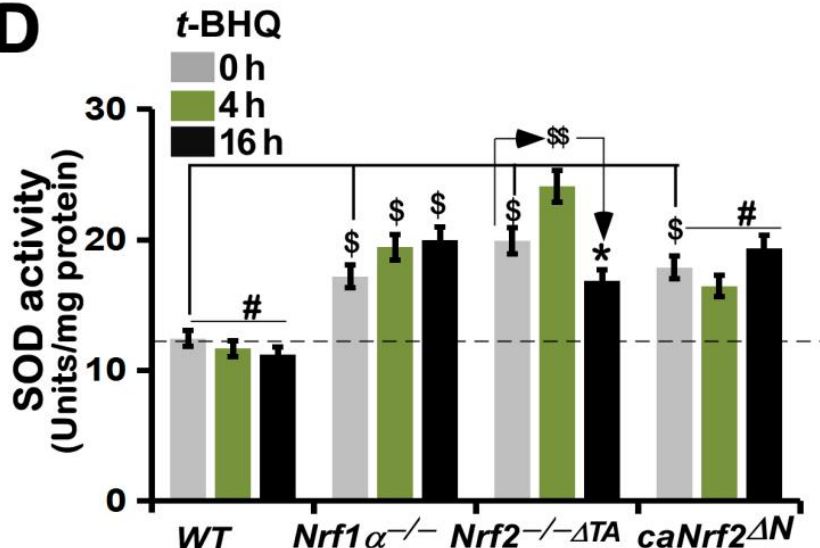

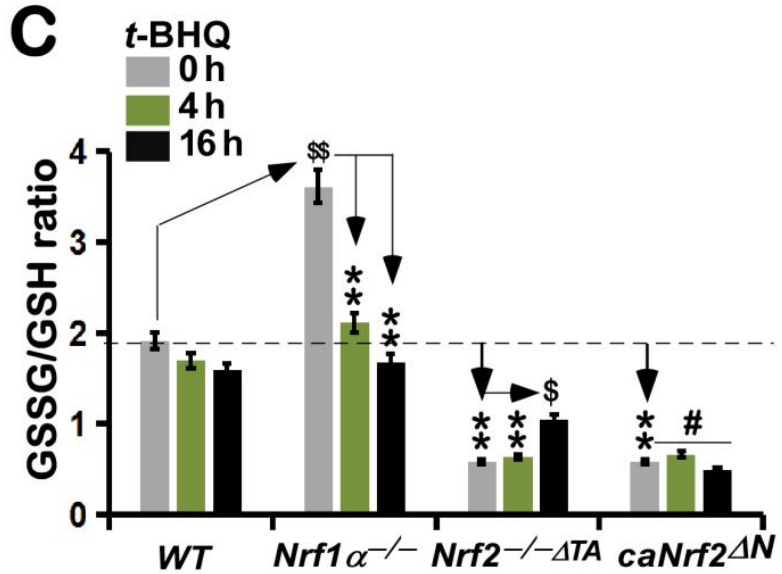
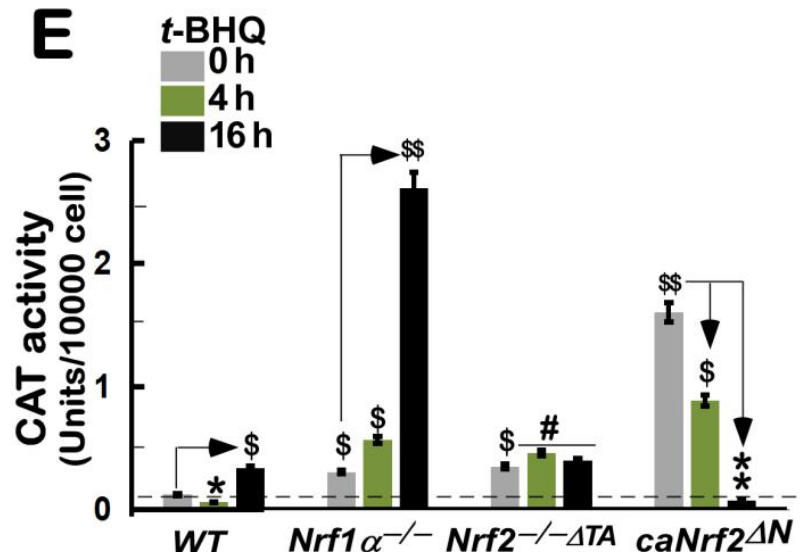

Figure 5. Altered levels of ROS, GSSG and GSH, along with ROS-scavenging activity of SOD and CAT, induced by $t B H Q$.

(A) Experimental cells of $W T, N r f 1 \alpha^{-/}, N r f 2^{-/-\Delta T A}$ and caNrf2 $2^{\Delta N}$ were allowed for treatment with $50 \mu \mathrm{M} t \mathrm{BHQ}$ or not for different time periods (i.e. $0,4,16 \mathrm{~h}$ ). Thereafter, the cells were subjected to a flow cytometry analysis of intracellular ROS by the DCFH-DA fluorescent intensity. The resulting data were further analyzed by FlowJo 7.6.1 software, as shown in the column charts (B). Statistic significances were also calculated as described in "Materials and Methods", with significant increases $(\$ \$, p<0.01)$, and significant decreases $\left({ }^{*} P<0.05 ;{ }^{*} P<0.01\right)$, respectively, in addition to the non-significance (\#). (C) The intracellular GSH and GSSH levels, together with two ROS-scavenging activities of SOD (D) and CAT (E), were measured according to the introduction of relevant kit manufacturers. The statistic significances were also determined as described above. 

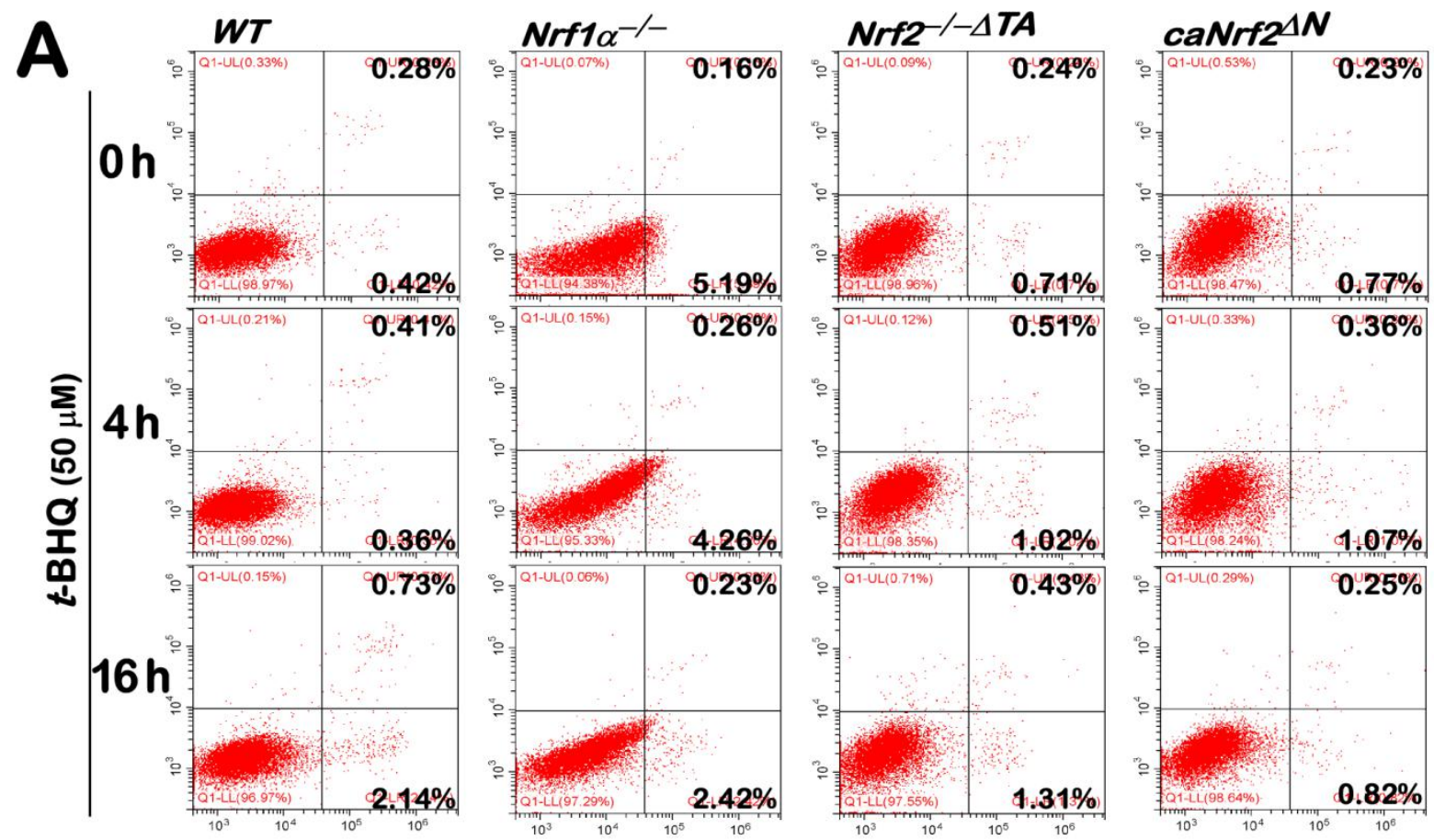

B

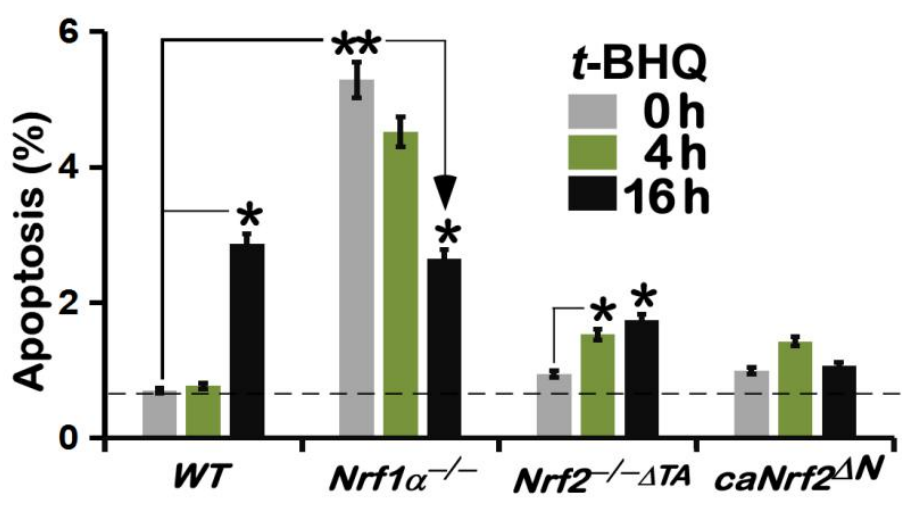

$\mathbf{C}$

MT1E-ARE1

5'-ctGCggaCTCA-3'

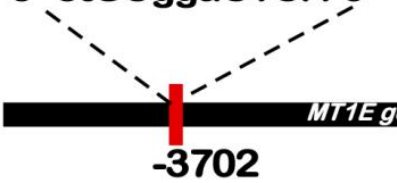

MT1E-ARE2

5'-acTGACaatGCac,-3'
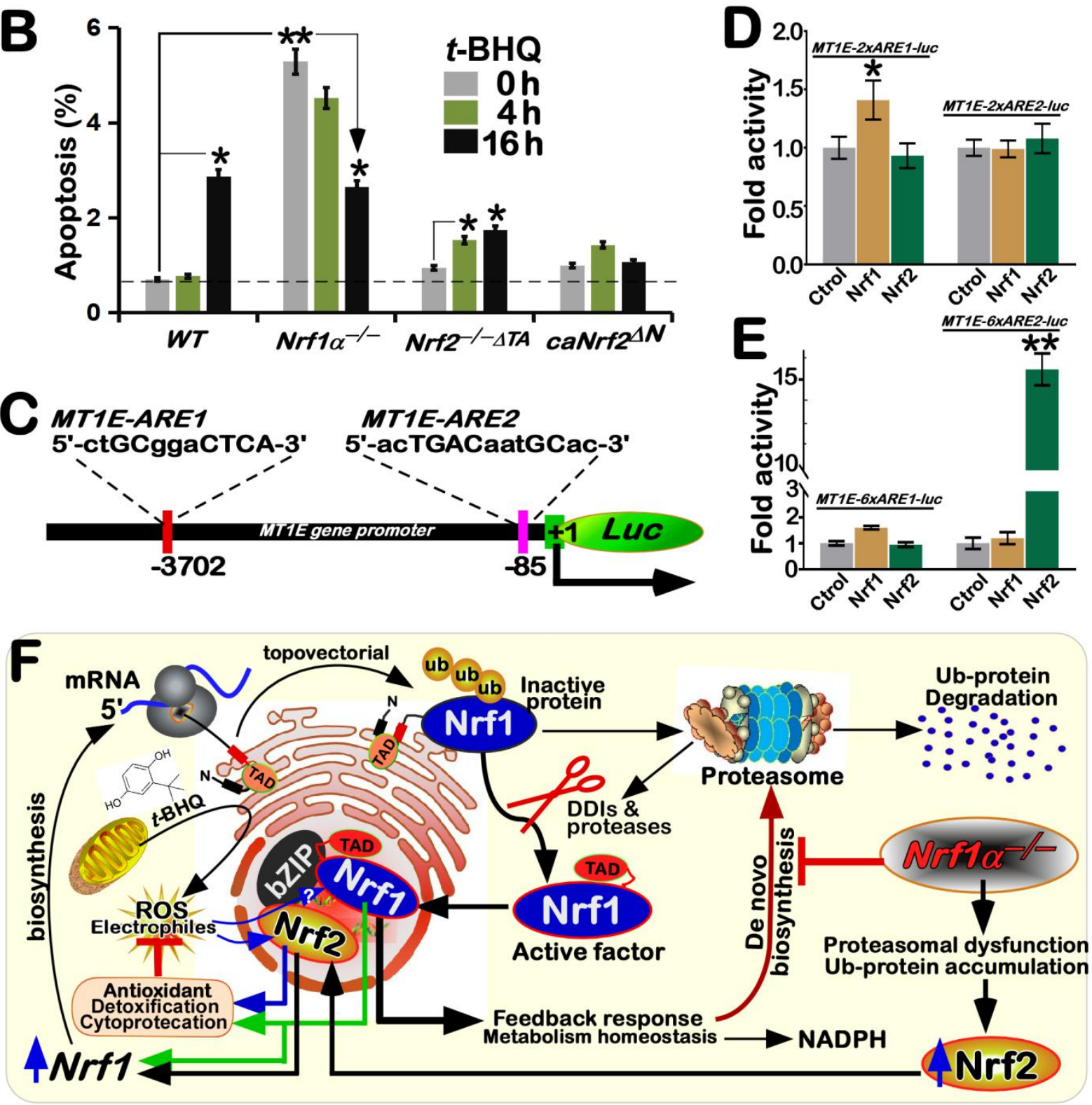
bioRxiv preprint doi: https://doi.org/10.1101/2021.06.05.447190; this version posted June 5, 2021. The copyright holder for this preprint (which was not certified by peer review) is the author/funder. All rights reserved. No reuse allowed without permission.

Figure 6. Nrf1 is more potent than Nrf2 at mediating the putative cytoprotective response to $t$-BHQ.

(A) Distinct genotypic cell lines of $W T, N r f 1 \alpha^{-/-}, N r f 2^{-/-\Delta T A}$ and caNrf2 ${ }^{\Delta N}$ were or were not treated with $50 \mu \mathrm{M} t \mathrm{BHQ}$ for different lengths of time. Subsequently, the cells were incubated with a binding buffer containing Annexin V-FITC and propidium iodide (PI) for $15 \mathrm{~min}$, before being subjected to the flow cytometry analysis of apoptosis. (B) The final data were shown by the column charts. The statistic significances were also determined, with significant increases $\left({ }^{*} P<0.05\right.$; $\left.{ }^{* *} P<0.01\right)$. (C) Two putative ARE sequences were shown in the location of the MT1E gene promotor region. (D \& E) Distinct copies of those two ARE-sequences from the MT1E gene were allowed for driving relevant luciferase reporter genes. Each of ARE-driven reporters, together with expression constructs for Nrf1, Nrf2 or an empty plasmid, were transfected into WT cells for the indicated times. Then the cells were allowed for recovery from transfection, before the luciferase activity was measured, as shown graphically. The statistical determination was indicated with significant increases $\left({ }^{*} P<0.05 ;{ }^{* *} P<0.01\right)$. (F) A proposed model is provided to give a better explanation of distinctive yet cooperative roles of Nrf1 and Nrf2 in synergistically regulating antioxidant cytoprotective genes. Of note, Nrf1 can act as a brake control for Nrf2's functionality to be confined within a certain extent, albeit its transcriptional expression is also positively regulated by Nrf2. 